\title{
ONE-DIMENSIONAL LONG-RANGE DIFFUSION LIMITED AGGREGATION II: THE TRANSIENT CASE
}

\author{
GIDEON AMIR, OMER ANGEL, AND GADY KOZMA
}

\begin{abstract}
We examine diffusion-limited aggregation for a one-dimensional random walk with long jumps. We achieve upper and lower bounds on the growth rate of the aggregate as a function of the number of moments a single step of the walk has. In this paper we handle the case of transient walks.
\end{abstract}

\section{Introduction}

Diffusion-limited aggregation (DLA for short) is a random growth model constructed as follows. Start with a single particle in space. Each subsequent particle performs a random walk "from infinity", until it hits any previous particle. It is then frozen and added to the aggregate at the last site it visited before hitting the aggregate. Precise definitions are included below. We refer the reader to part I [AABK] of this project for a history of the subject, and to part III [A] for some additional results.

The topic of these papers is one-dimensional long-range DLA. The following theorem was stated in part I (precise definitions are included in §2).

Theorem 1. Let $R$ be a symmetric random walk with step distribution satisfying $\mathbb{P}\left(\left|R_{1}-R_{0}\right|=k\right)=(c+o(1)) k^{-1-\alpha}$. Let $D_{n}$ be the diameter of the $n$ particle aggregate. Then almost surely:

(i) If $\alpha>3$, then $n-1 \leq D_{n} \leq C n+o(n)$, where $C$ is a constant depending only on the random walk.

(ii) If $2<\alpha \leq 3$, then $D_{n}=n^{\beta+o(1)}$, where $\beta=\frac{2}{\alpha-1}$.

(iii) If $1<\alpha<2$ then $D_{n}=n^{2+o(1)}$.

(iv) If $\frac{1}{3}<\alpha<1$ then

$$
\begin{gathered}
n^{\beta+o(1)} \leq D_{n} \leq n^{\beta^{\prime}+o(1)} \\
\text { where } \beta=\max \left(2, \alpha^{-1}\right) \text { and } \beta^{\prime}=\frac{2}{\alpha(2-\alpha)} \text {. } \\
\text { (v) If } 0<\alpha<\frac{1}{3} \text { then } D_{n}=n^{\beta+o(1)} \text {, where } \beta=\alpha^{-1} \text {. }
\end{gathered}
$$

See Figure 1 for the various regimes described in Theorem 1. In the regime $\alpha \in\left(\frac{1}{3}, 1\right)$, our results do not allow us to determine precisely the rate of growth, we conjecture that the lower bound gives the correct behaviour, and have therefore indicated it in the figure in red. Part I focused mainly on the recurrent case, namely $\alpha>1$. In this paper we focus on the transient case, namely $\alpha<1 / 3$ and $\alpha \in[1 / 3,1)$.

The most interesting feature of the graph is of course the phase transitions: at 3,2 and in at least one unknown point in $\left[\frac{1}{3}, 1\right]$, probably at $\frac{1}{2}$. We discussed the phase transitions at 3 and 2 in the introduction of part I quite thoroughly, so we will 


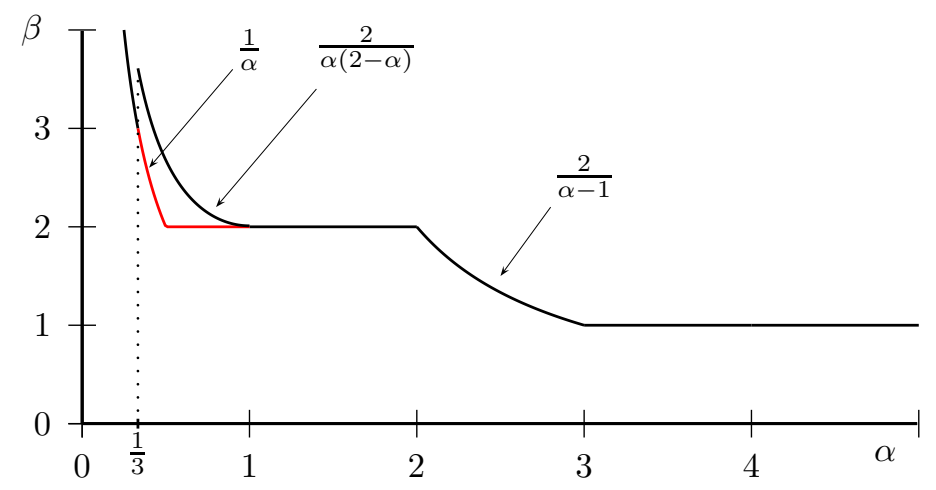

Figure 1. If the random walk $R$ has $\alpha$ finite moments, then the diameter of the resulting $n$-particle aggregate grows as $n^{\beta}$.

not repeat this here. Let us reiterate one point already made in part I nonetheless: it seems as if there is no change in the behaviour when passing from the recurrent regime to the transient regime (at $\alpha=1$ ). If our conjecture is correct then the growth of the aggregate is uniformly $n^{2+o(1)}$ throughout the interval $\left[\frac{1}{2}, 2\right]$ and one cannot tell from the aggregate the difference between recurrent and transient random walks. Note that these are not even quite the same processes on both sides - in the transient case one needs to condition on the particles hitting the aggregate, which makes the "gluing measure" quite different. Even if our conjecture is false, our upper bound still shows that the transition is smooth, hence the phase transition at $\alpha=1$, if it exists at all, is very tame.

\subsection{Proof ideas}

The key is that the growth of the aggregate does not come by gradual accretion but rather by isolated large jumps (in fact this holds for all $\alpha<3$, see part I for details). Hence to understand the behaviour of $A_{n}$ one needs to consider events whose probability is of the order of $1 / n$ - about one such event will happen in the construction of $A_{n}$, and this one rare event will dominate the growth of the aggregate.

We will show below that the probability that the aggregate $A_{n}$ grows by at least $m$ in one step is approximately

$$
\frac{n m^{-\alpha}}{\operatorname{Cap} A_{n}}
$$

where Cap $A_{n}$ is the capacity of the aggregate with respect to our random walk (see $\S 1.3$ for precise definitions). The reason that the capacity appears is that in order to define what does it mean for a particle to perform "a random walk from infinity" we have to condition on the particle hitting the set. This conditioning gives the factor of the capacity in the denominator, and is also the reason that the transient case $0<\alpha<1$ is more difficult than the recurrent case discussed in part I. Understanding the capacity requires more detailed knowledge about the structure of the aggregate. 
If the largest single jump gives the diameter, then we should have $\operatorname{diam} A_{n} \simeq m$ for the $m$ that corresponds to probability $\frac{1}{n}$. Solving $n m^{-\alpha} /$ Cap $A_{n}=1 / n$ gives

$$
\operatorname{diam} A_{n} \simeq\left(\frac{n^{2}}{\operatorname{Cap} A_{n}}\right)^{1 / \alpha}
$$

so an upper bound for the capacity gives a lower bound for the diameter and vice versa. There are two simple upper bounds for the capacity of a general finite set. The first is $\operatorname{Cap} A_{n} \leq n$ which gives $\operatorname{diam} A_{n} \gtrsim n^{1 / \alpha}$. The second bound is Cap $A_{n} \leq c\left(\operatorname{diam} A_{n}\right)^{1-\alpha}$ (see Lemma 8) which gives diam $A_{n} \gtrsim n^{2}$. This gives the lower bounds for $\alpha<1$ in Theorem 1 .

To test how good these two lower bounds are, consider a discrete, self-similar Cantor set. It turns out that up to constants, such a Cantor set has maximal possible capacity with respect to its diameter and number of particles (see Theorem 9 on page 12). This is surprising when one compares this to the structure of the harmonic measure on the fractal which is complicated and involves various exponents which, generally speaking, are not known. See Carleson [C85] and Makarov [M98] for analysis of Cantor-like sets, and the beautiful general results of Makarov [M89] and Jones and Wolff [JW88]. See also [B99].

Now, if the aggregate were to behave similarly, then it would have nearly maximal possible capacity which would lead to the minimal possible diameter. This would imply that the third phase transition is at $\frac{1}{2}$. If, on the other hand, the aggregate has a dense core with a few additional far away particles which do not affect the capacity significantly, we would get a much lower capacity, and hence higher diameter. In that case, the third phase transition could even be at 1 .

One regime where we can make this "Cantor set vs. heavy core" dichotomy precise, and so derive matching upper bounds on the diameter, is when the walk has less than $\frac{1}{3}$ moments. In this regime the walk makes enormous jumps - by time $n$ of the aggregation we expect a jump of size at least $n^{3}$, so a particle will be glued at large distance from the rest of the aggregate. Assume such a jump has happened, and call the resulting particle $b_{1}$. Let $b_{2}$ be the first time a particle coming from infinity hits $\left\{b_{1}\right\}$. Continuing this way we identify a subset $B$ in the aggregate consisting of $b_{1}$ and its descendents. A calculation then shows that the subset $B$ grows very similar to a DLA - the affect of the rest of the aggregate is only a time-change which does not affect the actual shape too much.

Since such large jumps occur at many scales, we think of DLA as a stochastic Cantor set, where remote parts are identically distributed, (though not identical, as in the usual Cantor set). The crux of the argument is in making this precise and proving this. We show that the probability that the existing part $A \backslash B$ affects the growth of the subset $B$ or vice versa at all is quite small. This is not true for $\alpha>\frac{1}{3}$. For such $\alpha$, the amount of interaction between the two parts, while small, is not zero, and we do not know how to control it.

Of course, the actual proof entails a few complications. Since we lose constants in various places, it is not enough to divide $A_{n}$ into 2 parts and show that they are roughly identically distributed. We therefore divide $A$ into $\log n$ parts, show that (with high probability) they are identically distributed and that the capacity of the union is close to the sum of their capacities (again, because they are far away) this gives the formula

$$
\operatorname{Cap} A_{n} \simeq(\log n) \operatorname{Cap} A_{n / \log n}
$$


Now the loss of constants is immaterial and we get Cap $A_{n} \geq n^{1-o(1)}$, and so the upper bound on the diameter. Another complication is that it seems difficult to work with the expectation of $\sum 1 / \operatorname{Cap} A_{n}$ (this sum turns out to be the relevant quantity to study) because it is difficult to control unusual events which might make this sum large. Thus instead of expectations we work with medians and quantiles, and you will see them strewn all over the proof.

The approach could also give improved bounds for some $\alpha>\frac{1}{3}$. In fact, the condition required for the argument to apply is roughly that $n^{2}\left(\operatorname{diam} A_{n}\right)^{\alpha-1} \lesssim 1$ (see Lemma 24 on page 23 below). This means that if $\operatorname{diam} A_{n} \gtrsim n^{2 /(1-\alpha)}$ then our argument will go through, leading to a contradiction as the capacity grows linearly while the diameter is still large. In short, for $\alpha \in\left(\frac{1}{3}, 1\right)$ it is possible to show that $\operatorname{diam} A_{n} \leq n^{2 /(1-\alpha)+o(1)}$ using essentially the same argument as we use for $\alpha<\frac{1}{3}$. This estimate is better than our stated upper bound in the interval $\left[\frac{1}{3}, \frac{1}{2}(3-\sqrt{5})\right]$ (approximately 0.38). Nevertheless, we will not prove this estimate, as we do not believe it contributes additional understanding to the problem. We remark, though, that adding this extra result would remove the discontinuity in the graph of our upper bound, making it continuous (but not monotone).

\subsection{What should you read?}

This paper is more technical than part I, with a particular emphasis on the case of $\alpha<\frac{1}{3}$. If you are only interested in a tasting of the ideas used, your best option is probably to switch to part I and read $\S 3$ there which handles one particular transient random walk whose analysis is simpler and more geometric than other cases.

In Section 2 we derive a general formula for the gluing measure in the transient case. Section 3 then discusses the lower bound $D_{n} \geq n^{\beta+o(1)}$ which holds for all $0<\alpha<1$. Finally Section 4 gives the proof for the case of $\alpha<\frac{1}{3}$. This paper is best read sequentially as each section depends on all previous ones.

Finally, note that there is a part III [A] which discusses the infinite aggregate $A_{\infty}=\bigcup_{n=1}^{\infty} A_{n}$.

\subsection{Notations}

For a subset $A \subset \mathbb{Z}$ we denote by $\operatorname{diam} A$ the diameter of $A$, namely $\max (A)-$ $\min (A)$. If $x \in \mathbb{Z}$ we will denote by $\rho(x, A)$ the point-to-set distance, namely $\min _{y \in A}|x-y|$.

Throughout this paper we denote by $R=\left(0=R_{0}, R_{1}, \ldots\right)$ a random walk on $\mathbb{Z}$. We denote by $p_{x, y}=p_{0, y-x}$ the probability that $R$, when starting from $x$ will go to $y$ in the first step. For a given random walk $R$ and set $A$, let $T_{A}$ be the hitting time of $A$, defined as

$$
T_{A}=\inf \left\{n>0 \text { s.t. } R_{n} \in A\right\} .
$$

Note that $T_{A}>0$ even if the random walk starts in $A$. Denote by $E_{A}(x)$ the escape probability from $A$ i.e. $\mathbb{P}_{x}\left(T_{A}=\infty\right.$ ) (where $\mathbb{P}_{x}$ denotes the law of the random walk started at $x)$. Denote by $E_{A}^{*}(x)$ the escape probability for the reversed random walk (we will usually consider symmetric random walks, in which case $E_{A}=E_{A}^{*}$ ). 
Define the capacity of a set by

$$
\operatorname{Cap} A=\sum_{a \in A} E_{A}^{*}(x)
$$

See $\S 3.1$ for a detailed discussion of the capacity.

Denote by $G(x, y)$ the Green function of $R$ defined by

$$
G(x, y)=\sum_{n=0}^{\infty} \mathbb{P}_{x}\left(R_{n}=y\right)
$$

Since $G$ is translation invariant we will often abbreviate $G(x, 0)$ as $G(x)$ and then $G(x, y)=G(x-y)$. It turns out that regular behaviour of the Green function is the most important property for our analysis.

By $C$ and $c$ we denote constants which depend only on the law of the walk $R$ but not on the other parameters involved. The same holds for constants hidden within the notations $O$ and $o$. In particular, we will usually not use them again the way they were used in theorem 1 that is, as random constants. Generally $C$ and $c$ might take different values at different places, even within the same formula. Normally, $C$ will pertain to constants which are "big enough" and $c$ to constants which are "small enough". By $X \approx Y$ we mean $c X<Y<C X$. By $X \ll Y$ we mean that $X=o(Y) .\lfloor x\rfloor$ will denote the integer value of $x$. By $\simeq$ and $\lesssim$ we do not mean anything in particular; these are only used for the heuristic discussion in the introduction.

\section{Limits of gluing measure}

The random walks we consider will usually satisfy the following:

Definition. We say that a symmetric random walk is an $\alpha$-walk $(\alpha<1)$ if $p_{0, x} \approx$ $|x|^{-1-\alpha}$.

(As usual $\approx$ means "bounded between two constants".) For such walks the Green function has the following behaviour:

Lemma 2. Let $R$ be an $\alpha$-walk. Then its corresponding corresponding Green function satisfies

$$
G(x) \approx|x|^{\alpha-1} .
$$

Proof. We base our proof on a result of Bass \& Levin [BL02]. Let us recall the statement of Theorem 1.1 ibid. Bass \& Levin do not assume that the walk is translation invariant, and their result holds in any dimension, though we specialize to $d=1$. Given numbers $w_{x y}$ satisfying $w_{x y} \approx|x-y|^{-1-\alpha}$ and $w_{x y}=w_{y x}$, define a Markov chain $R$ by its transition probabilities

$$
\mathbb{P}_{x}\left(R_{1}=y\right)=\frac{w_{x y}}{\sum_{z} w_{x z}} .
$$

We may therefore take $w_{x y}=p_{x, y}$. A minor inconvenience is that Bass \& Levin assume that $w_{x x}=0$. Let us therefore assume it for our walk $R$ for a while, and remove this assumption in the end. Their results then state that

$$
\mathbb{P}_{x}\left(R_{n}=y\right) \approx \min \left(n^{-1 / \alpha}, n|x-y|^{-1-\alpha}\right)
$$

with the exception that the lower bound does not hold for $n=1$ and $x=y$ (again, because $w_{x x}=0$ ). 
Summing (2) over $n$ immediately gives (1). Thus we need only remove the restriction $p_{x, x}=0$. But this only changes $G$ by a constant, i.e. if for some $R$ we have $p_{x, x}>0$ then we can define a walk $R^{\prime}$ using

$$
\mathbb{P}\left(R_{1}^{\prime}=x\right)= \begin{cases}\frac{p_{0, x}}{1-p_{x, x}} & x \neq 0 \\ 0 & x=0 .\end{cases}
$$

and get that the corresponding Green function, $G^{\prime}$, satisfies $G^{\prime}(x)=G(x) / p_{x, x}$. This finishes the lemma.

Let us remark that the results of Bass \& Levin are not restricted to the transient case, they hold also for the recurrent case. However, it is not as straightforward to get information on the harmonic potential from the estimate of the heat kernel, as it is for the Green function in the transient case.

While on this topic we might remark that a number of results in the nonreversible settings are known. The results of Williamson [W68] are quite general, essentially requiring only that the walk $R$ is in the domain of attraction of an $\alpha$ stable process, but they are restricted to $\frac{1}{2}<\alpha<1$. Le Gall and Rosen have some results for general $\alpha$ [GR91, Proposition 5.2].

Let $A \subset \mathbb{Z}$ be some set. For a point $x \notin A$, we are interested in the event $\left\{R_{T_{A}-1}=x\right\}$, that is the event that $x$ is the last point visited before hitting $A$, and so is glued. Since the random walks we are interested in are transient we must condition on $T_{A}<\infty$. Define

$$
\mu_{y}(x, a)=\mathbb{P}_{y}\left(R_{T_{A}}=a, R_{T_{A}-1}=x \mid T_{A}<\infty\right) .
$$

We are interested in the limit

$$
\mu(x, a)=\mu(x, a ; A)=\lim _{y \rightarrow \infty} \mu_{y}(a, x),
$$

if it exists, as well as its integrated version

$$
\mu(x)=\mu(x ; A)=\sum_{a \in A} \mu(x, a) .
$$

The existence of these limits is strongly related to the existence of the harmonic measure from infinity, which is defined as the limit of $\sum_{x} \mu(x, a)$ (see Lemma 5 below).

It is possible that the harmonic measure does not exist. An example can be constructed by examining a random walk supported on a very sparse set, for example let $\mathbb{P}\left(R_{1}=2^{2^{n}}\right) \approx 1 / n^{2}$ and 0 otherwise. As this is somewhat off-topic we will not provide a proof that this is indeed an example. Instead, the following theorem characterises walks for which the harmonic measure exists, and relates the harmonic measure to the escape probabilities.

Theorem 3. The following are equivalent for a transient irreducible random walk on $\mathbb{Z}$ :

(i) For any finite $A$, the harmonic measure exists and equals the normalized escape probabilities.

(ii) The harmonic measure exists for some finite $A$ with $|A|>1$,

(iii) For any a the Green function satisfies $\lim _{|x| \rightarrow \infty} \frac{G(x+a)}{G(x)}=1$. 
When the conditions of this theorem hold we say that $R$ has a harmonic measure.

Recall that the escape probabilities are denoted by $E_{A}(x)=\mathbb{P}_{x}\left(T_{A}=\infty\right)$, and that this definition is non-trivial also for starting points in $A$. Recall also that $E_{A}^{*}(a)$ is the analogous probability for the reversed random walk (for symmetric random walk the two are the same). The capacity of a set $A$ is defined as $\operatorname{Cap}(A)=$ $\sum_{a \in A} E_{A}^{*}(a)$.

Note that the result is stated also for asymmetric walks even though we are mostly interested in the symmetric case. Further it can be easily generalised to the case of $\mathbb{Z}^{d}$ - the only difference being that in Clause (ii) we must require that $A$ is not a subset of any affine subspace of $\mathbb{R}^{d}$.

Part of the proof $(($ iii $) \Longrightarrow($ i) $)$ follows the proof of P26.2 of [S76] which does the same for random walks in $\mathbb{Z}^{3}$.

Proof of Theorem 3. Let $\Pi$ be the $A$-indexed matrix, with $\Pi(a, b)=\mathbb{P}_{a}\left(R_{T_{A}}=b\right)$. Let $G(x-\cdot)$ be the row vector $\{G(x-a)\}_{a \in A}$. We use the following identity for the unnormalized hitting measure $H_{A}(x, a)=\mathbb{P}_{x}\left(R_{T_{A}}=a\right)$

Proposition 4. $H_{A}(x, a)=\sum_{z \in A} G(x-z)(I-\Pi)(z, a)$.

A proof can be found in [S76, P25.1]. For the reader's convenience, let us indicate a simple probabilistic argument (Spitzer's proof is more analytic in nature). Examine the random walk restricted to $A$ : The probability to hit $A$ at $a$ is the expected number of times $a$ is visited minus the number of visits that are not the first visit to $A$. The former is $G(x, a)$; the latter can be partitioned according to the location of $z$ : the previous visit to $A$.

(iii) $\Longrightarrow$ (i): By Proposition 4 and our assumption (iii),

$$
\lim _{|x| \rightarrow \infty} \frac{H_{A}(x, a)}{G(x)}=\lim _{|x| \rightarrow \infty} \sum_{z \in A} \frac{G(x-z)}{G(x)}(I-\Pi)(z, a) \stackrel{(\mathrm{iii})}{=} \sum_{z \in A}(I-\Pi)(z, a) .
$$

The sum of column $a$ in $I-\Pi$ is $E_{A}^{*}(a)$, so

$$
\lim _{|x| \rightarrow \infty} \frac{H_{A}(x, a)}{G(x)}=E_{A}^{*}(a) \quad \forall a \in A .
$$

Summing over $a$ gives

$$
\mathbb{P}_{x}\left(T_{A}<\infty\right)=G(x) \cdot \operatorname{Cap}(A) \cdot(1+o(1)) .
$$

Which gives the required:

$$
\begin{aligned}
\lim _{|x| \rightarrow \infty} \mathbb{P}_{x}\left(R_{T_{A}}=a \mid T_{A}<\infty\right) & =\lim _{|x| \rightarrow \infty} \frac{H(x, a)}{\mathbb{P}_{x}\left(T_{A}<\infty\right)} \\
& =\lim _{|x| \rightarrow \infty} \frac{H(x, a)}{G(x) \operatorname{Cap}(A)(1+o(1))}=\frac{E_{A}^{*}(a)}{\operatorname{Cap}(A)} .
\end{aligned}
$$

Since (i) $\Rightarrow$ (ii) is obvious, it remains to show that (ii) implies (iii). For any finite $A, I-\Pi$ is strictly diagonally dominant and therefore invertible (by the Levy-Desplanques theorem, see e.g. [HJ85, Theorem 6.1.10]). Thus if the harmonic measure exists (which means, by definition, that $\lim _{|x| \rightarrow \infty} \mathbb{P}\left(R_{T_{A}}=a \mid T_{A}<\infty\right.$ ) 
exists), then

$$
\begin{aligned}
\lim _{|x| \rightarrow \infty} \frac{G(x-a)}{\mathbb{P}_{x}\left(T_{A}<\infty\right)} & =\lim _{|x| \rightarrow \infty} \sum_{z \in A} \frac{H_{A}(x, z)}{\mathbb{P}_{x}\left(T_{A}<\infty\right)}(I-\Pi)^{-1}(z, a) \\
& =\sum_{z \in A} \mu(z)(I-\Pi)^{-1}(z, a)
\end{aligned}
$$

and in particular the limit on the left-hand side exists. Hence also the limit of $G(x-a) / G(x-b)$ exists, for all $a, b \in A$.

Assume without loss of generality that $0, b \in A$ and then the limit of $G(x-$ $b) / G(x)$ exists. Since the limit is the same for positive and negative $x$, and since $0<G(x) \leq G(0)$ is bounded, the limit must be $1-$ if it were smaller than 1 then $G$ would diverge exponentially in the direction of $b$ while if it were larger than 1 then $G$ would diverge exponentially in the other direction.

Clearly $\frac{G(x-b)}{G(x)} \rightarrow 1$ implies that $\frac{G(x-k b)}{G(x)} \rightarrow 1$ for any $k$. To show that $\frac{G(x+a)}{G(x)} \rightarrow 1$ for general $a$ we need irreducibility for the first time. Let $T$ be the first time at which $R_{T} \equiv a(\bmod b)$, and note that $T$ is a.s. finite. Fix $\varepsilon>0$ and let $N=N(a, \varepsilon)$ be such that $\mathbb{P}\left(\left|R_{T}-a\right|>N\right)<\varepsilon$. Take $M=M(a, \varepsilon, N)$ large enough that whenever $|k b|<N$ and $x>M$ we have $G(x+k b)>(1-\varepsilon) G(x)$.

By the Markov property at $T$, for $|x|>M$.

$$
\begin{aligned}
G(x+a) & =\sum_{y \equiv a \bmod b} \mathbb{P}\left(R_{T}=y\right) G(x+a-y) \\
& \geq \sum_{\substack{|y|<N \\
y \equiv a \bmod b}} \mathbb{P}\left(R_{T}=y\right)(1-\varepsilon) G(x) \\
& \geq(1-\varepsilon)^{2} G(x) .
\end{aligned}
$$

Since $\varepsilon$ was arbitrary, $\lim \inf _{x} G(x+a) / G(x) \geq 1$. Since the same holds for $-a$ this completes the proof.

We now come to the key connection between escape probabilities and the DLA gluing measure.

Lemma 5. If the harmonic measure for a transient random walk $R$ exists, then the limit $\mu=\lim _{y} \mu_{y}$ exists and

$$
\mu(x, a)=\frac{p_{x, a} E_{A}^{*}(x)}{\operatorname{Cap}(A)} .
$$

We remind the reader that $p_{x, a}$ is the probability of making a single step from $x$ to $a$.

Proof. Define $F(z)=\mathbb{P}_{z}\left(T_{x}<T_{A}\right)$ to be the probability that $x$ is hit from $z$ before $A$ (and in particular $\left.T_{x}<\infty\right)$. Start a random walk at $y$. One can partition visits to $x$ according to the previous visited site of $A \cup\{x\}$ to get

$$
G(y, x)=G(y-x)=F(y)+G(y-x) F(x)+\sum_{a \in A} G(y-a) F(a) .
$$

On the other hand, the expected number of visits to $x$ before visiting $A$ is given by $\frac{F(y)}{1-F(x)}$. Let $M_{x, a}$ be the event that the random walk hits $A$ by a step from $x$ to $a$, 
then

$$
\begin{aligned}
\mathbb{P}_{y}\left(M_{x, a}\right) & =\frac{F(y) p_{x, a}}{1-F(x)} \\
& =p_{x, a} \cdot\left(G(y-x)-\frac{1}{1-F(x)} \sum_{a \in A} G(y-a) F(a)\right) .
\end{aligned}
$$

Using (3) to approximate $\mathbb{P}_{y}\left(T_{A}<\infty\right)=G(y) \operatorname{Cap}(A)(1+o(1))$ and condition (iii) of Theorem 3 to eliminate the ratio of Green function values as $y \rightarrow \infty$ one finds

$$
\mathbb{P}_{y}\left(M_{x, a} \mid T_{A}<\infty\right)=\frac{p_{x, a}}{\operatorname{Cap}(A)} \cdot\left(1-\frac{1}{1-F(x)} \sum_{a \in A} F(a)\right)(1+o(1))
$$

(here $o(1)$ is as $|y| \rightarrow \infty$ ).

Next we relate this to the reversed escape probability from $x$. Run a reversed random walk from $x$. Paths that hit $A$ at $a$ can be broken into some number of loops where the walk returns to $x$ followed by a path from $x$ to $a$ that avoids $A \cup\{x\}$. The reverse of a loop rooted at $x$ is just a loop; the reverse of the last segment is one of the terms contributing to $F(a)$. Thus we have

$$
\mathbb{P}_{x}^{*}(\text { hit } A \text { at } a)=\frac{F(a)}{1-F(x)} .
$$

Summing over $a \in A$ gives the probability of hitting $A$ and so

$$
\mathbb{P}_{y}\left(M_{x, a} \mid T_{A}<\infty\right)=\frac{p_{x, a}}{\operatorname{Cap}(A)} E_{A}^{*}(x)(1+o(1)) .
$$

We are finally ready to give the formal definition of the DLA process for transient random walks:

Definition 1. Let $R$ be a transient random walk with harmonic measure on $\mathbb{Z}$. The $D L A$ process with respect to $R$ is a Markov chain of random sets $A_{1}=\{0\}, A_{2}, \ldots$ such that for any finite $A \subset \mathbb{Z}$, and $x \in \mathbb{Z} \backslash A$ and any $n>0$,

$$
\mathbb{P}\left(A_{n+1}=A \cup\{x\} \mid A_{n}=A\right)=\sum_{a \in A} \mu(x, a ; A)
$$

where $\mu$ is defined by (4). We denote $D_{n}=\operatorname{diam} A_{n}$ and $\mathscr{F}_{n}$ the minimal $\sigma$-field generated by $A_{1}, \ldots, A_{n}$.

\section{Inequalities}

Recall the gluing formula (4). It turns out that the most crucial term for understanding the DLA growth is the capacity $\operatorname{Cap}(A)$ appearing in the denominator. In $\S 3.1$ we will prove estimates for the capacity of any finite set in $\mathbb{Z}$. In $\S 3.3$ we will use these estimates to get first bounds on the diameter of the aggregates. Henceforth, all walks are assumed to be symmetric, and we will not remind this fact again.

\subsection{General inequalities for capacity}

Before embarking on estimates of the capacity of our aggregate, let us recall some general facts about this notion. The capacity of a set can be defined in two equivalent ways. The first (which we used to prove (4)) is as the sum of the escape 
probabilities. The second is a variational definition:

$$
\operatorname{Cap}(A)=\sup _{\psi \in \mathscr{P}(A)} \frac{1}{\|\psi * G\|_{\infty}},
$$

where $\mathscr{P}(A)$ is the set of all probability measures on $A$ (or simply all $\psi: A \rightarrow[0,1]$ with $\left.\sum \psi(a)=1\right), G$ is the Green function of the walk and $\psi * G$ denotes the usual convolution on $\mathbb{Z}$ i.e.

$$
(\psi * G)(x):=\sum_{y} G(x-y) \psi(y) .
$$

See [S76, P25.10] for the equivalence of the two definitions. There is a unique measure $\psi_{0}$ satisfying the supremum which is called the equilibrium charge. It satisfies that $\psi_{0} * G$ is in fact constant over $A$ and is characterized by this fact. In fact, the equilibrium charge is the normalized escape probabilities, namely

$$
\psi_{0}(x)=\frac{\mathbb{P}_{x}\left(T_{A}=\infty\right)}{\sum_{a \in A} \mathbb{P}_{a}\left(T_{A}=\infty\right)} .
$$

See [S76, chap. 25] for an overview and proof of the equivalence of the two definitions of $\psi_{0}$. We start with an application of capacity to show that starting far from a set suffices for a good probability of avoiding it.

Lemma 6. Let $R$ be an $\alpha$-walk. For any $\lambda>0$

$$
\inf \left\{E_{A}(x): \rho(x, A)>\lambda \operatorname{diam} A \text { and } x>\max (A)\right\}>0 \text {. }
$$

Proof. By translation invariance and since $E_{A}(x)$ is monotone in $A$, we may assume $A=[-k, 0]$ for some $k$ (and then $\operatorname{diam} A=k$ ). Define

$$
\varphi(\lambda)=\inf _{k, w>\lambda k}\left\{E_{[-k, 0]}(w)\right\} .
$$

We will first prove $\varphi\left(\lambda_{0}\right)>0$ for some $\lambda_{0}$ large enough. Let $\psi$ be the equilibrium charge of $[-k, 0]$, and let $p=\psi * G$. By [S76, T25.1],

$$
1-E_{[-k, 0]}(w)=\frac{p(w)}{p(0)} \quad \forall w \notin[-k, 0] .
$$

(Note that $p(\cdot)$ is constant on $[-k, 0]$, so 0 is not singled out here.) Let us first assume that $w \geq \lambda_{0} k$ for some $\lambda_{0}$ sufficiently large. Then we can write (using $G(x) \approx|x|^{\alpha-1}$, Lemma 2)

$$
\begin{aligned}
p(w) & =\sum_{i=0}^{k} \psi(-i) G(w+i) \leq C \sum_{i=0}^{k} \psi(-i)\left(\lambda_{0} k\right)^{\alpha-1} \\
& \leq C \lambda_{0}^{\alpha-1} \sum_{i=0}^{k} \psi(-i)(1+i)^{\alpha-1} \leq C \lambda_{0}^{\alpha-1} \sum_{i=0}^{k} \psi(-i) G(i) \\
& =C \lambda_{0}^{\alpha-1} p(0)
\end{aligned}
$$

and we see that for $\lambda_{0}$ sufficiently large, independent of $k, E_{[0, k]}(w) \geq \frac{1}{2}$, and so $\varphi\left(\lambda_{0}\right) \geq \frac{1}{2}$.

Next, fix some $\lambda>0$. Let $w>\lambda k$, and let $I$ be the reflection of $[-k, 0]$ about $w$, i.e. the interval $[2 w, 2 w+k]$. By symmetry we have

$$
\mathbb{P}_{w}\left(T_{[-k, 0]}<T_{I}\right) \leq \frac{1}{2} .
$$


However, after $T_{I}$ (if it is finite), the probability to escape to infinity is at least $\varphi(2 \lambda)$. Thus we get

$$
\varphi(\lambda) \geq \frac{1}{2} \varphi(2 \lambda) .
$$

Since $\varphi\left(\lambda_{0}\right)>0$ and $\varphi$ is increasing, this implies $\varphi(\lambda)>0$ for all $\lambda>0$.

Lemma 7. For an $\alpha$-walk $R$, the capacity of any finite set $A$ satisfies

$$
\operatorname{Cap}(A) \geq c|A|^{1-\alpha} \text {. }
$$

If moreover $A \subset d \mathbb{Z}$, then

$$
\operatorname{Cap}(A) \geq c \min \left\{|A|,(d|A|)^{1-\alpha}\right\} .
$$

Proof. We use the variational definition of the capacity. Examine the uniform probability measure $\psi_{u}$. We get

$$
\operatorname{Cap}(A) \geq\left\|\psi_{u} * G\right\|_{\infty}^{-1} .
$$

By $G(x) \approx|x|^{\alpha-1}$ (Lemma 2) we get

$$
\begin{aligned}
\left(\psi_{u} * G\right)(x) & =\sum_{y} G(x-y) \psi(y) \leq \sum_{y}\left(C|x-y|^{\alpha-1}\right) \cdot \frac{1}{|A|} \\
& \leq \frac{1}{|A|} \sum_{i=1}^{|A|}\left(C i^{\alpha-1}\right) \leq C|A|^{\alpha-1}
\end{aligned}
$$

and the first claim is proved. If $A$ is contained in an arithmetic progression, the same argument gives

$$
\left(\psi_{u} * G\right)(x) \leq C|A|^{-1}+C(d|A|)^{\alpha-1},
$$

where the first term comes from the contribution of $y=x$.

Lemma 8. For an $\alpha$-walk $R$ the capacity of any finite set $A$ satisfies

$$
\operatorname{Cap}(A) \leq C(\operatorname{diam} A)^{1-\alpha} .
$$

Proof. It follows directly from the variational definition (5) that $\operatorname{Cap}(A)$ is increasing in $A$ (see [S76, P25.11 (b)] for a different but equally simple argument). Filling in the holes in $A$ and translating to 0 we get $\operatorname{Cap}(A) \leq \operatorname{Cap}([0, \operatorname{diam} A])$. To estimate the capacity of an interval we use the definition of capacity as a sum of escape probabilities and write

$$
\operatorname{Cap}([0, n]) \leq 2 \sum_{i=1}^{n / 2} \mathbb{P}_{0}\left(\left|R_{1}\right|>i\right)
$$

i.e. we bound the escape probability simply by the probability to exit the set in the first step. Since $R$ is an $\alpha$-walk, this implies

$$
\operatorname{Cap}([0, n]) \leq C \sum i^{-\alpha} \leq C n^{1-\alpha} .
$$

To demonstrate that the combination of these two bounds is sharp, let us prove the result promised in the introduction concerning the capacity of the Cantor set. While this is a bit of a digression, some of our results indicate that the DLA aggregate is self-similar, and is built up of several copies of smaller aggregates, well separated in $\mathbb{Z}$. Thus the following serves as further indication that the capacity of our aggregate $A_{n}$ is indeed roughly $\min \left\{n,\left(\operatorname{diam} A_{n}\right)^{1-\alpha}\right\}$, and consequently the diameter grows as $n^{\min \{2,1 / \alpha\}+o(1)}$ also for $\alpha \in[1 / 3,1]$. 
Theorem 9. Let $A_{n}$ be the discrete Cantor set

$$
A_{n}:=\left\{i=0, \ldots, 3^{n}-1: \forall j=0, \ldots, n-1\left\lfloor i / 3^{j}\right\rfloor \not \equiv 1 \quad \bmod 3\right\} .
$$

Let $R$ be an $\alpha$-walk. Then

$$
\text { Cap } A_{n} \geq c \min \left\{2^{n}, 3^{n(1-\alpha)}\right\} / n .
$$

Further, if $\alpha \neq-\log _{3} 2+1$ then the estimate may be slightly improved to

$$
\text { Cap } A_{n} \geq c(\alpha) \min \left\{2^{n}, 3^{n(1-\alpha)}\right\} .
$$

Note that by lemma 8 Cap $A_{n} \leq 3^{n(1-\alpha)}$ and since $\left|A_{n}\right|=2^{n}$ we have Cap $A_{n} \leq$ $2^{n}$. Hence (for $\alpha$-walks) the theorem is precise up to at most the logarithmic factor $n$.

Proof. Let $\psi$ be the equilibrium charge over $A_{n}$. Then

$$
\frac{1}{\operatorname{Cap} A_{n}}=(\psi * G)(x) \quad \forall x \in A_{n}
$$

and therefore

$$
\begin{aligned}
\frac{1}{\operatorname{Cap} A_{n}} & =2^{-n} \sum_{x \in A_{n}}(\psi * G)(x)=2^{-n} \sum_{x \in A_{n}} \sum_{y \in A_{n}} \psi(y) G(x-y)= \\
& =2^{-n} \sum_{y \in A_{n}} \psi(y) \sum_{x \in A_{n}} G(x-y) \\
& \leq C 2^{-n} \sum_{y \in A_{n}} \psi(y) \sum_{x \in A_{n}}|x-y|^{\alpha-1}
\end{aligned}
$$

where in the last inequality we used Lemma 2. Now for every $y \in A_{n}$ it is easy to calculate

$$
\begin{aligned}
\sum_{x \in A_{n}}|x-y|^{\alpha-1} & =\sum_{k=0}^{n-1} \sum_{x: 3^{k} \leq|x-y| \leq 3^{k+1}}|x-y|^{\alpha-1} \leq \\
& \leq \sum_{k=0}^{n-1} 3^{k(\alpha-1)} \#\left\{x:|x-y| \leq 3^{k+1}\right\} \leq C \sum_{k=0}^{n-1} 3^{k(\alpha-1)} 2^{k} \leq \\
& \leq C n \max \left(2^{n} 3^{n(\alpha-1)}, 1\right) .
\end{aligned}
$$

Further, if $\alpha \neq-\log _{3} 2+1$ then the last inequality may be strengthened and we get

$$
\sum_{x \in A_{n}}|x-y|^{\alpha-1} \leq C(\alpha) \max \left(2^{n} 3^{n(\alpha-1)}, 1\right) \quad \forall y \in A_{n}
$$

Plugging (7) and (8) into (6) gives the two parts of the theorem, respectively (remember that $\left.\sum \psi(y)=1\right)$.

Monotonicity of the capacity implies that adding a point to a set will increase its capacity. The next lemma gives a nice formula for the exact increment in capacity. Recall that for a set $A$ and point $x, E_{A}(x)$ is the escape probability starting at $x$. We also define $E_{A}^{\prime}(x)=E_{A \cup\{x\}}(x)$, i.e. the probability that $x$ is also avoided by the random walk. 
Lemma 10. Let $A$ be a set with finite capacity with respect to a transient symmetric random walk, let $x$ be a point outside $A$, and let $A^{\prime}=A \cup\{x\}$. Then

$$
\operatorname{Cap}\left(A^{\prime}\right)-\operatorname{Cap}(A)=E_{A}(x) E_{A}^{\prime}(x)
$$

and in particular, $\operatorname{Cap}\left(A^{\prime}\right)-\operatorname{Cap}(A) \in\left[E_{A}^{\prime}(x)^{2}, E_{A}(x)^{2}\right]$.

Proof. From the representation of the capacity as a sum of escape probabilities we have $\operatorname{Cap}\left(A^{\prime}\right)-\operatorname{Cap}(A)=E_{A}^{\prime}(x)-\sum_{a \in A}\left(E_{A}(a)-E_{A^{\prime}}(a)\right)$. Since the event of escaping from $A^{\prime}$ is contained in the event of escaping from $A$, for $a \in A$ we have,

$$
E_{A}(a)-E_{A^{\prime}}(a)=\mathbb{P}_{a}\left(T_{x}<\infty, T_{A}=\infty\right) .
$$

Random walk paths that escape from $A$ and hit $x$, a.s. visit $x$ only finitely many times. Breaking each path at the last visit to $x$ we have

$$
E_{A}(a)-E_{A^{\prime}}(a)=\sum_{\substack{\gamma: a \rightarrow x \\ \text { outside A }}} \mathbb{P}(\gamma) \mathbb{P}_{x}\left(T_{A^{\prime}}=\infty\right)=E_{A}^{\prime}(x) \sum_{\substack{\gamma: a \rightarrow x \\ \text { outside A }}} \mathbb{P}(\gamma)
$$

where $\mathbb{P}(\gamma)$ denotes the probability that the random walk follows the path $\gamma$ until its end. By reversing each path $\gamma$ :

$$
E_{A}(a)-E_{A^{\prime}}(a)=E_{A}^{\prime}(x) \mathbb{P}_{x}(R \text { hits } A \text { at } a),
$$

since the sum is just the probability that the walk starting at $x$ hits $A$ at $a$. Summing over $a \in A$ :

$$
\operatorname{Cap}\left(A^{\prime}\right)-\operatorname{Cap}(A)=E^{\prime}(x)-E_{A}^{\prime}(x) \mathbb{P}_{x}\left(T_{A}<\infty\right)=E_{A}^{\prime}(x) E_{A}(x) .
$$

\subsection{Capacity and DLA}

Lemma 11. Let $R$ be an $\alpha$-walk. Let $A_{n}$ be a corresponding DLA process. Then there exists some $\delta>0$ such that

$$
\mathbb{P}\left(\operatorname{Cap}\left(A_{n+1}\right)-\operatorname{Cap}\left(A_{n}\right)>\delta \mid \mathscr{F}_{n}\right) \geq \frac{c n}{\operatorname{Cap}\left(A_{n}\right)^{1 /(1-\alpha)}} .
$$

Here $c$ and $\delta$ may depend on the walk, but not on $n$.

Proof. Since $A_{n}$ is an arbitrary set of $n$ elements, let us denote it by $A$ and its capacity by $\kappa$. For some $\delta$ to be determined, let $S_{\delta}=A \cup\left\{x: E_{A}(x) E_{A}^{\prime}(x) \leq \delta\right\}$ be the set of points whose addition to $A$ will increase the capacity by less than $\delta$. The first step is to show that $S_{\delta}$ is small, in the sense that at most half the points in any interval of length at least $\lambda \kappa^{1 /(1-\alpha)}$ are in $S_{\delta}$, where $\lambda$ is some constant to be determined. The scale $\kappa^{1 /(1-\alpha)}$ is chosen so that the capacity of the whole interval is comparable to $\kappa$.

To see this, observe first that $E_{A}(x) E_{A}^{\prime}(x)$ is decreasing in $A$. Let $D=\left\{k_{1}, \ldots\right.$, $\left.k_{|D|}\right\}$ be an arbitrary subset of $S_{\delta}$. Then

$$
\begin{aligned}
\operatorname{Cap}(D) & \leq \operatorname{Cap}(A \cup D) \\
& =\operatorname{Cap}(A)+\sum_{i=1}^{|D|} \operatorname{Cap}\left(A \cup\left\{k_{1}, \ldots, k_{i}\right\}\right)-\operatorname{Cap}\left(A \cup\left\{k_{1}, \ldots, k_{i-1}\right\}\right) \\
& =\kappa+\sum_{i=1}^{|D|} E_{A \cup\left\{k_{1}, \ldots, k_{i-1}\right\}}\left(k_{i}\right) E_{A \cup\left\{k_{1}, \ldots, k_{i}\right\}}\left(k_{i}\right) \leq \kappa+|D| \delta
\end{aligned}
$$


Fix $d=\kappa^{\alpha /(1-\alpha)}$. For an interval $I$ of length $\lambda \kappa^{1 /(1-\alpha)}$, we apply this to $D=$ $I \cap(d \mathbb{Z}) \cap S_{\delta}$. Since $|D| \leq|I| / d=\lambda \kappa$, we find from (9) that $\operatorname{Cap}(D) \leq(1+\delta \lambda) \kappa$.

On the other hand, from Lemma 7 we have

$$
(1+\delta \lambda) \kappa \geq \operatorname{Cap}(D) \geq c_{1} \min \left\{|D|,(d|D|)^{1-\alpha}\right\} .
$$

Assume $|D|>|I| / 2 d=\lambda \kappa / 2$, then we have either $1+\delta \lambda>c_{1} \lambda / 2$, or else $1+\delta \lambda>$ $c_{1}(\lambda / 2)^{1-\alpha}$. Neither of these hold for $\lambda$ large enough and $\delta$ small enough, giving a contradiction. Since there is nothing special about points that are $0(\bmod d)$, we find that $S_{\delta}$ contains at most half of every congruency class in in $I$, and thus at most half of $I$. At this point we fix the values of $\lambda$ and $\delta$ to satisfy the requirement just stated, and from now on we treat them as constants and will hide them inside $c$-s.

To complete the proof, note using the gluing formula (4) that

$$
\begin{array}{r}
\mathbb{P}\left(\operatorname{Cap}\left(A_{n+1}\right)-\operatorname{Cap}\left(A_{n}\right)>\delta \mid \mathscr{F}_{n}\right) \geq \sum_{x \notin S_{\delta}} \mathbb{P}\left(A_{n+1}=A_{n} \cup\{x\}\right) \\
\stackrel{(4)}{=} \sum_{x \notin S_{\delta}} \sum_{a \in A_{n}} \frac{p_{x, a} E_{A_{n}}(x)}{\operatorname{Cap} A_{n}} \geq \sum_{a \in A_{n}} \sum_{x \notin S_{\delta}} \frac{c|x-a|^{-\alpha-1} \delta}{\kappa}
\end{array}
$$

(where in the last inequality we used that for $x \in S_{\delta}$ we also have $E_{A}(x)>\delta$.) For every $a \in A$ we estimate its contribution to the sum by applying the above with $I=\left[a, a+\lambda \kappa^{1 /(1-\alpha)}\right]$. We get

$$
\sum_{x \notin S_{\delta}}|x-a|^{-\alpha-1} \geq \sum_{x \in I \backslash S_{\delta}} c|I|^{-\alpha-1} \geq \frac{|I|}{2} \cdot c|I|^{-\alpha-1}=c \kappa^{-\alpha /(1-\alpha)} .
$$

Returning the term $c \delta / \kappa$ and summing over the $n$ elements of $A$ gives the claim.

With this result in place, we are ready to prove our first non-trivial bounds on the capacity of the DLA generated by an $\alpha$-walk.

Lemma 12. Let $R$ be an $\alpha$-walk, and $\left(A_{n}\right)$ the associated $D L A$. Then almost surely there exists some $c$ such that

$$
\operatorname{Cap}\left(A_{n}\right)>c n^{\frac{2-2 \alpha}{2-\alpha}}
$$

for all large enough n. Consequently, this bounds holds a.s. for all $n$ with a random $c$.

Proof. The key is that a long as the capacity is small, the probability that it grows is not too small. Let $X_{n}$ be the events

$$
X_{n}:=\left\{\operatorname{Cap}\left(A_{n}\right)<n^{\frac{2-2 \alpha}{2-\alpha}}\right\} \cap\left\{\operatorname{Cap}\left(A_{n+1}\right)-\operatorname{Cap}\left(A_{n}\right) \leq \delta\right\}
$$

where $\delta$ is from Lemma 11 . We can bound $\mathbb{P}\left(X_{n} \mid \mathscr{F}_{n}\right)$ by 0 if $\operatorname{Cap}\left(A_{n}\right)$ is large, and so using Lemma 11 we have

$$
\mathbb{P}\left(X_{n} \mid \mathscr{F}_{n}\right)<1-\frac{c n}{n^{2 /(2-\alpha)}}=1-c_{2} n^{-\frac{\alpha}{2-\alpha}} .
$$

Since $\operatorname{Cap}\left(A_{n}\right)$ is increasing in $n$, it suffices to prove the claim for $n=2^{k}$. Let $B_{k}$ be the (bad) event that too many $X_{n^{-}}$s happened between $2^{k}$ and $2^{k+1}$ namely

$$
B_{k}:=\left\{\#\left\{X_{n}: 2^{k} \leq n<2^{k+1}\right\} \geq 2^{k}\left(1-\frac{1}{2} c_{2} 2^{-(k+1) \frac{\alpha}{2-\alpha}}\right)\right\} .
$$


By (10) the $X_{n}$ are stochastically dominated by i.i.d. variables and hence the probability of $B_{k}$ may be estimated by standard inequalities for such sums. We get

$$
\mathbb{P}\left(B_{k}\right) \leq \exp \left(-c 2^{k-(k+1) \frac{\alpha}{2-\alpha}}\right) \leq \exp \left(-c 2^{k \frac{2-2 \alpha}{2-\alpha}}\right) .
$$

Therefore $\sum \mathbb{P}\left(B_{k}\right)$ converges and a.s. only a finite number of the $B_{k}$ occur. The lemma will follow once we show that for some $c>0$ sufficiently small $\neg B_{k}$ implies $\operatorname{Cap}\left(A_{2^{k+1}}\right) \geq c 2^{k(2-2 \alpha) /(2-\alpha)}$.

If for any $2^{k} \leq n<2^{k+1}$ we have $\operatorname{Cap}\left(A_{n}\right) \geq n^{(2-2 \alpha) /(2-\alpha)}$ then

$$
\operatorname{Cap}\left(A_{2^{k+1}}\right)>\operatorname{Cap}\left(A_{n}\right)>\left(2^{k}\right)^{\frac{2-2 \alpha}{2-\alpha}}=c\left(2^{k+1}\right)^{\frac{2-2 \alpha}{2-\alpha}}
$$

Otherwise, $\neg B_{k}$ implies that

$$
\operatorname{Cap}\left(A_{n+1}\right)-\operatorname{Cap}\left(A_{n}\right)>\delta \quad \text { for at least } c 2^{k \frac{2-2 \alpha}{2-\alpha}} n \text {-s. }
$$

This of course implies

$$
\operatorname{Cap}\left(A_{2^{k+1}}\right) \geq c \delta 2^{k \frac{2-2 \alpha}{2-\alpha}}
$$

which was to be proved.

\subsection{From capacity to diameter}

We will now use the various estimates on the escape probabilities and capacity of $A_{n}$ proved in the previous section to get bounds on the the diameter of $A_{n}$. This section is analogous to $\S 3$ in part I.

Theorem 13. For an $\alpha$-walk $R$ one has

$$
\operatorname{diam}\left(A_{n}\right) \geq\left(\frac{n^{2}}{\operatorname{Cap} A_{n}}\right)^{1 / \alpha} \text { infinitely often }
$$

and

$$
\operatorname{diam}\left(A_{n}\right) \geq\left(\frac{c n^{2}}{(\log \log n) \operatorname{Cap} A_{n}}\right)^{1 / \alpha} \text { for all } n \text { sufficiently large }
$$

both with probability 1.

Proof. Fix some $m, D$ and set $A$ with $|A|=m$, and examine the growth probability $\mathbb{P}\left(\operatorname{diam} A_{m+1}>D \mid A_{m}=A\right)$. If $\operatorname{diam} A>D$, then this probability is simply 1 . Otherwise, suppose that $x$ was glued to some $a \in A$. If $|x-a|>2 D$, then $\operatorname{diam} A_{m+1}>D$ (in fact this even implies that diam $A_{m+1} \geq 2 D$ ) but it also implies that $d(x, A)>D \geq \operatorname{diam} A$. Thus we can use Lemma 6 to estimate $E_{A}(x)>c$. We get

$$
\begin{aligned}
& \mathbb{P}\left(\operatorname{diam} A_{m+1}>D \mid A_{m}=A\right) \geq \sum_{\substack{a \in A \\
x:|x-a|>2 D}} \mu(x, a)= \\
& \stackrel{(4)}{=} \sum_{a \in A} \sum_{x:|x-a|>2 D} \frac{E_{A}(x) p_{x, a}}{\operatorname{Cap}(A)} \geq \sum_{a \in A} \frac{c \mathbb{P}\left(\left|R_{1}\right|>2 D\right)}{\operatorname{Cap}(A)} \geq \frac{c m D^{-\alpha}}{\operatorname{Cap}(A)} .
\end{aligned}
$$

Applying this for $D_{m}=\left(4 m^{2} / \operatorname{Cap} A_{m}\right)^{1 / \alpha}$, we get that the events $\operatorname{diam} A_{m+1}>$ $D_{m}$ stochastically dominate a sequence of independent Bernoulli trials with probability $c / m$. By the Borel-Cantelli Lemma, a.s. infinitely often diam $A_{m+1}>D_{m}>$ $\left(\frac{m^{2}}{\operatorname{Cap} A_{m}}\right)^{1 / \alpha}$. 
For the second part of the theorem, we apply (11) with

$$
D_{m}=\left(\frac{c_{2} m^{2}}{(\log \log m) \operatorname{Cap} A_{m}}\right)^{1 / \alpha} \text {. }
$$

For some $c_{2}$ sufficiently small, we get that the events diam $A_{m+1}>D_{m}$ dominate a sequence of independent Bernoulli trials with probability $(4 \log \log m) / m$. Thus

$$
\begin{aligned}
& \mathbb{P}\left(\exists m \in\left[\frac{1}{2} n, n\right) \text { s.t. } \operatorname{diam} A_{m+1}>D_{m}\right) \geq 1-\prod_{m=n / 2}^{n-1}\left(1-\frac{4 \log \log m}{m}\right) \\
& \geq 1-\left(1-\frac{4 \log \log n}{n}\right)^{n / 2} \geq 1-\frac{c}{\log ^{2} n} .
\end{aligned}
$$

Existence of such $m$ implies that $\operatorname{diam} A_{n} \geq D_{m} \geq \frac{1}{4} D_{n}$. By Borel-Cantelli, this a.s. holds for $n=2^{k}$ for all large enough $k$. Moving from $n=2^{k}$ to general $n$ only loses a constant (again by the monotonicity of the capacity and diameter), and the second claim of the theorem is proved.

Corollary 14. Under the assumptions of Theorem 13,

$$
\begin{aligned}
& \operatorname{diam}\left(A_{n}\right) \geq c \max \left(n^{1 / \alpha}, n^{2}\right) \text { infinitely often } \\
& \operatorname{diam}\left(A_{n}\right) \geq \max \left(\left(\frac{n}{\log \log n}\right)^{1 / \alpha}, \frac{n^{2}}{\log \log n}\right) \text { for all } n \text { sufficiently large. }
\end{aligned}
$$

Proof. The lower bound $n^{1 / \alpha}$ follows from using Theorem 13 with the trivial bound Cap $A_{n} \leq n$. The lower bound $n^{2}$ follows from applying the bound Cap $A_{n} \leq$ $C\left(\operatorname{diam} A_{n}\right)^{1-\alpha}$ (Lemma 8$)$ and moving terms around.

We now turn to our current best upper bound for the diameter for $\alpha \in(1 / 3,1)$. The following is a companion to Theorem 13, with reversed direction for the inequalities.

Theorem 15. For any symmetric random walk $R$ with $\mathbb{P}_{0}\left(\left|R_{1}\right|>x\right) \leq C x^{-\alpha}$ (and in particular any $\alpha$-walk) one has a.s.

$$
\operatorname{diam}\left(A_{n}\right)=o\left(n(\log n)^{1+\varepsilon} \max _{m<n} \frac{m}{\operatorname{Cap} A_{m}}\right)^{1 / \alpha} .
$$

Proof. As in the proof of Theorem 13, let $m, D$ be integers and $A$ a set of size $m$. Condition on $A_{m}=A$ and assume $A_{m+1}$ was constructed by gluing some $x$ at some $a \in A$. Then clearly $\operatorname{diam} A_{m+1}-\operatorname{diam} A_{m} \leq|x-a|$. Hence we can write

$$
\begin{aligned}
& \mathbb{P}\left(\operatorname{diam} A_{m+1}-\operatorname{diam} A_{m}>D \mid A_{m}=A\right) \leq \sum_{\substack{a \in A \\
|x-a|>D}} \mu(x, a) \\
&=\sum_{\substack{a \in A \\
|x-a|>D}} \frac{E_{A}(x) p_{x, a}}{\operatorname{Cap} A} \leq \sum_{a \in A} \frac{\mathbb{P}_{0}\left(\left|R_{1}\right|>D\right)}{\operatorname{Cap} A} \leq \frac{C m D^{-\alpha}}{\operatorname{Cap} A} .
\end{aligned}
$$

Here we do not need Lemma 6 or some analog of it, because we simply estimate $E_{A}(x) \leq 1$. 
Denote therefore $D_{m, k}=\left(2^{-k} n m / \operatorname{Cap} A_{m}\right)^{1 / \alpha}$ and examine the event

$$
L_{m, k}:=\left\{\operatorname{diam} A_{m+1}-\operatorname{diam} A_{m}>D_{m, k}\right\} .
$$

By (12) we have that $\mathbb{P}\left(L_{m, k} \mid A_{m}\right) \leq C 2^{k} / n$. In other words, $L_{m, k}$ (for each fixed $k$ and $m=1, \ldots, n)$ are stochastically dominated by i.i.d. Bernoulli trials with probabilities $C 2^{k} / n$ so we expect at most $2^{k}$ such events. Standard estimates for Bernoulli trials give

$$
\mathbb{P}\left(\text { at least } \lambda 2^{k} L_{m, k} \text { occurred }\right) \leq C\left(\begin{array}{c}
n \\
\left\lceil\lambda 2^{k}\right\rceil
\end{array}\right)\left(\frac{C 2^{k}}{n}\right)^{\left\lceil\lambda 2^{k}\right\rceil} \leq\left(\frac{C}{\lambda}\right)^{\left\lceil\lambda 2^{k}\right\rceil}
$$

which holds for all $\lambda>0$ and all $k$.

Let $\mathscr{G}_{n}$ be the event that for all $k$, no more than $\lambda 2^{k}$ of the $L_{m, k}$ occur. Notice that when $\lambda 2^{k}<1$ this means no $L_{m, k}$ occur, and since $L_{m, k}$ is increasing in $k$, no $L_{m, k^{\prime}}$ occur for any $k^{\prime}<k$ either. By (13), if $\lambda$ is sufficiently large then $\mathbb{P}\left(\mathscr{G}_{n}\right)>1-C / \lambda$, since it suffices to check the events for $k$ with $\lambda 2^{k} \geq 1 / 2$.

For any given $m$, if $k$ is maximal so that $\operatorname{diam} A_{m+1}-\operatorname{diam} A_{m} \leq D_{m, k-1}$, then $L_{m, k}$ occurs, and so

$$
\operatorname{diam} A_{m+1}-\operatorname{diam} A_{m} \leq \sum_{k} \mathbf{1}_{L_{m, k}} D_{m, k-1} .
$$

Assuming $\mathscr{G}_{n}$ we can sum over $m$ to get

$$
\begin{aligned}
\operatorname{diam} A_{n}-\operatorname{diam} A_{n / 2} & =\sum_{m=n / 2}^{n-1} \operatorname{diam} A_{m+1}-\operatorname{diam} A_{m} \\
& \leq \sum_{m=n / 2}^{n} \sum_{k} \mathbf{1}_{L_{m, k}} D_{m, k-1} \\
& \leq \sum_{k: 2^{k} \lambda \geq 1} \lambda 2^{k} \max _{m<n} D_{m, k-1} \\
& =\lambda\left(n \max _{m<n} \frac{m}{\operatorname{Cap} A_{m}}\right)^{1 / \alpha} \sum_{k: 2^{k} \lambda \geq 1} 2^{k(1-1 / \alpha)} \\
& \leq C\left(\lambda n \max _{m<n} \frac{m}{\operatorname{Cap} A_{m}}\right)^{1 / \alpha} .
\end{aligned}
$$

Now fix $\lambda=c_{1}(\log n)^{1+\varepsilon}$ for some $c_{1}$ sufficiently small. Applying that $\mathbb{P}\left(\mathscr{G}_{n}\right) \leq C / \lambda$ for $n=2^{l}$ we see that $\mathscr{G}_{2^{l}}$ happens for all $l>l_{0}$ so we get

$$
\operatorname{diam} A_{2^{l}} \leq \operatorname{diam} A_{2^{l_{0}}}+\sum_{i=l_{0}}^{l} C\left(c_{1} 2^{i}\left(\log 2^{i}\right)^{1+\varepsilon} \max _{m<2^{i}} \frac{m}{\operatorname{Cap} A_{m}}\right)^{1 / \alpha}
$$

Passing from $n=2^{l}$ to general $n$ only costs a constant and we get

$$
\operatorname{diam} A_{n} \leq \operatorname{diam} A_{2^{l_{0}}}+C\left(c_{1} n(\log n)^{1+\varepsilon} \max _{m<n} \frac{m}{\operatorname{Cap} A_{m}}\right)^{1 / \alpha} .
$$

Since $c_{1}$ is arbitrary, the claim follows.

Corollary 16. Let $R$ be an $\alpha$-walk. Then

$$
\operatorname{diam} A_{n} \leq n^{2 / \alpha(2-\alpha)+o(1)}
$$


Proof. By Lemma 12 we have

$$
\text { Cap } A_{n}>c n^{(2-2 \alpha) /(2-\alpha)}
$$

for some random $c$, with probability 1 . Using this in Theorem 15 gives

$$
\operatorname{diam} A_{n} \leq\left(n(\log n)^{1+\varepsilon} \max _{m<n} C m^{\alpha /(2-\alpha)}\right)^{1 / \alpha}=n^{2 / \alpha(2-\alpha)+o(1)} .
$$

\section{Less than $\frac{1}{3}$ moments}

In this section we will handle the case of $\alpha<\frac{1}{3}$. As already explained, we need to give an estimate for the capacity. This is theorem 17 below - the $\alpha<\frac{1}{3}$ clause of Theorem 1 is then an immediate corollary of it and of Theorem 15.

Theorem 17. Let $R$ be a random walk satisfying $\mathbb{P}\left(R_{1}=x\right) \approx x^{-1-\alpha}$ for $\alpha \in$ $\left(0, \frac{1}{3}\right)$. Then the DLA generated by $R$ a.s. satisfies $\operatorname{Cap}\left(A_{n}\right)=n^{1-o(1)}$.

Throughout most of our analysis in this section we aim to derive properties of the DLA at some time $n$, and to relate them to the DLA at time $\lfloor n / \log n\rfloor$ (here and below we write $n / \log n$, implicitly taking the integer part). Since $n$ is generally fixed, we frequently make it implicit.

\subsection{Continuous time}

Let us introduce continuous time. This is not strictly needed for our analysis, but does simplify some of the proofs. DLA in continuous time is defined as follows. We start with $A_{1}=\{0\}$. Given the aggregate $A_{t}$, each point $a \in A_{t}$ becomes active with rate 1 . When a point $a$ is activated, we start a random walk $\left(R_{i}\right)$ from $R_{0}=a$. If $R$ avoids $A_{t}$, then $R_{1}$ is added to $A_{t}$. The whole step is instantaneous (the speed of $R$ is infinite, if you like). If $\left(R_{i}\right)$ hits $A_{t}$ then $A$ remains unchanged. Let

$$
\tau(m):=\inf \left\{t:\left|A_{t}\right|=m\right\} .
$$

It is easy to conclude from the gluing formula (4) that the sequence $\left(A_{\tau(m)}\right)_{m}$ of sets visited by the process $A_{t}$ is the DLA process - indeed, once one conditions on an addition of a particle at time $t$, the probability that some $a \in A$ was the one activated is exactly $E_{A_{t}}(a) / \operatorname{Cap}\left(A_{t}\right)$ while the probability that the first step was to $x$ (conditioned on the random walk from $a$ escaping to infinity) is exactly $p_{x, a} E_{A_{t}}(x) / E_{A_{t}}(a)$, so one recovers the gluing formula.

From now on, we shall simply refer to the DLA in continuous time as DLA. We will also abbreviate $\tau=\tau(n)$ as $n$ will be fixed for long parts of the analysis. Since new points are added at $\operatorname{rate} \operatorname{Cap}\left(A_{t}\right) \in\left[1,\left|A_{t}\right|\right]$, it is clear that $\left|A_{t}\right|$ grows at most exponentially fast and so the process is well defined for all $t$. In light of Theorem 17, this is not too far from the truth.

\subsection{Split DLA}

The core of the argument is in introducing a process that allows us to analyse the dependency between distant parts of the aggregate. We will call this process split DLA (SDLA). This process is similar to DLA but contains two components that grow independently. In fact SDLA is not a single process, but a family of processes, though the dependence on the parameters $(n, D$ and $q)$ will be implicit. 
We will construct a coupling of DLA and SDLA for all values of $q$, which is then shown to have useful properties, while keeping the two aggregates equal up to time $\tau(n)$ for most values of $q$ (SDLA is also constructed in continuous time). In fact, we believe that w.h.p. the two processes are equal up to time $\tau(n)$ for all values of $q$, though proving that is not needed for our argument.

SDLA is defined in terms of two parameters. A carefully selected $D$ defined below, (16) (depending implicitly on $n$ ), and an integer $q \in \mathbb{N}$. The SDLA consists of two sets $S_{t}$ and $\widehat{S}_{t}$. Initially we have $S_{1}=\emptyset$ and $\widehat{S}_{1}=\{0\}$. The SDLA dynamics are very similar to DLA. Each of $S_{t}$ and $\widehat{S}_{t}$ evolves as an independent DLA in continuous time, with a small exception: Each time a random walk is started at a point of $\widehat{S}$, there is some probability that the first step $\left|R_{1}-a\right|$ of the random walk is large, namely greater than $D$. On the $q^{\text {th }}$ time that this happens, we add $R_{1}$ to $S_{t}$ (which up to that time has been empty), and we do not add it to $\widehat{S}_{t}$. This is done whether or not the random walk later hits $\widehat{S}_{t}$ or not. Similarly, "the $q^{\text {th }}$ time" counts initial steps greater than $D$ irrespectively of whether a point was added to $\widehat{S}$ or not.

While normally we keep the dependence on $q$ implicit, we will need it at some points. We will use $S_{t}^{q}$ and $\widehat{S}_{t}^{q}$ to denote the two parts of the SDLA when we wish to make this dependence explicit. The dependence on $D$ and $n$ is always implicit. When a random walk used in the DLA begins with a large jump, we say that a split occurs. We denote the time of the $q^{\text {th }}$ split by

$$
\beta_{q}=\inf \left\{t: S_{t}^{q} \neq \emptyset\right\} .
$$

This is the birth time of the $q$-SDLA. The birth point i.e. the first point in $S^{q}$ will be denoted by $b_{q}$. We will also care about the first time at which a split occurs starting from a point $a \in S_{t}^{q}$, and denote this by $\zeta_{q}$. Finally, to consider the time at which the parts of the SDLA reaches certain sizes, we use $\tau_{q}(m)$ and $\widehat{\tau}_{q}(m)$. We shall also consider the time at which the total size of the SDLA is $m$, denoted $\sigma(m)$.

In the next few sections we will investigate SDLA as an independent object. We will only return to the coupling of DLA and SDLA in $\S 4.7$. Nevertheless we might as well explain it roughly at this point, to give the reader some perspective. The coupling is very natural. Before the $q^{\text {th }}$ split of the DLA, we use the same activation times for the DLA and for the $\widehat{S}$ part of the SDLA, and the same random walks. They evolve the same. After the $q^{\text {th }}$ split, as long as $A_{t}=\widehat{S}_{t} \cup S_{t}$ and $\widehat{S}_{t} \cap S_{t}=\emptyset$ we again use the same activation times and the same random walks. Now there is no deterministic guarantee that they evolve the same, but we will see that, for $\alpha<\frac{1}{3}$ they indeed do, with high probability. Once one of these conditions is violated, we simply let them evolve independently (the exact condition for "separation" of the DLA and SDLA is a little different, see lemma 29 below, but the above serves as a good approximation).

\subsection{Splitting estimates}

We now choose the parameter $D$ for the SDLA. Define

$$
M_{n}:=\operatorname{Med}\left(\sum_{i=1}^{n} \frac{1}{\operatorname{Cap}\left(A_{\tau(i)}\right)} ; \frac{5}{6}\right) \quad M:=M_{n / \log n},
$$


where $\operatorname{Med}(X ; p)$ is the " $p$-Median" (quantile) of the variable $X$ i.e.

$$
\operatorname{Med}(X ; p)=\sup \{t: \mathbb{P}(X<t)<p\} .
$$

Note that points are added to $A$ at $\operatorname{rate} \operatorname{Cap}(A)$, so $\sum_{i=1}^{n} \frac{1}{\operatorname{Cap}\left(A_{\tau(i)}\right)}$ is an estimate for $\tau(n)$. With this $M$, define

$$
D:=\left(\frac{6 C_{1} n M}{\log n}\right)^{1 / \alpha}
$$

where $C_{1}$ is such that the random walk jump distribution satisfies $\mathbb{P}(|X|>t)<$ $C_{1} t^{-\alpha}$ for all $t$.

Note that $\operatorname{Cap}(A) \leq|A|$, and therefore $M \geq \sum_{1}^{n / \log n} i^{-1} \geq c \log n$. It follows that

$$
D \geq c n^{1 / \alpha} \text {. }
$$

We show below that this is not far from the truth: $M=n^{o(1)}$. The purpose of this definition of $D$ is to have both lower and upper bounds on the occurrence of such large jumps and in this way to control the branching of the SDLA.

Lemma 18. For $n$ sufficiently large, the probability that a DLA splits before (continuous) time $\min (2 M, \tau(n / \log n))$ is at most $\frac{1}{3}$.

Lemma 19. There exists a $c_{2}>0$ so that

$$
\mathbb{P}\left(A \text { splits fewer than } \log n \text { times by } \tau(n / 2) \text { and } \operatorname{Cap}\left(A_{\tau}\right)<c_{2} n / M\right) \leq C n^{-c} .
$$

Since the splits of DLA and SDLA are the same, up to the $q^{\text {th }}$ split, these lemmas give information also on SDLA. We will use them mainly for DLA, though.

We will see that $\tau(n / \log n)$ is typically less than $2 M$. Thus we argue that $A$ is likely to accumulate $n / \log n$ points without splitting, but very likely to split many times before accumulating $n / 2$ points, unless it has large capacity.

Proof of Lemma 18. Given $A_{t}$, the rate at which splits occur is the probability of a large random walk jump times $\left|A_{t}\right|$, namely $\left|A_{t}\right| \mathbb{P}(|X|>D) \leq\left|A_{t}\right| \cdot C_{1} D^{-\alpha}$. Using the definition of $D(16)$, as long as $|A|<n / \log n$ the rate of splits is at most $\frac{1}{6 M}$. Therefore the number of splits up to time $\min (2 M, \tau(n / \log n))$ is stochastically dominated by a Poisson process with rate $\frac{1}{6 M}$ and time interval $2 M$. It follows that the expected number of splits by time $\min (2 M, \tau(n / \log n))$ is at most $\frac{1}{3}$.

Proof of Lemma 19. The probability of a large random walk jump is at least $c D^{-\alpha}$, so the splitting rate is at least

$$
\left|A_{t}\right| c D^{-\alpha}=\frac{c(\log n)\left|A_{t}\right|}{n M} .
$$

The rate at which new points are added to $A_{t}$ is $\operatorname{Cap}\left(A_{t}\right)$. Thus the probability that a split occurs before a point is added to $A_{t}$ is at least

$$
\frac{c(\log n)\left|A_{t}\right|}{n M \operatorname{Cap}\left(A_{t}\right)} \text {. }
$$

With this $c$, fix $c_{2}=c / 20$. If $\left|A_{t}\right| \geq n / 4$ and $\operatorname{Cap}\left(A_{t}\right)<c_{2} n / M$ then the probability of a new split is at least $5(\log n) / n$. 
Let $X_{k}$ be the indicator of the event that a split occurs in the interval $(\tau(k), \tau(k+$ $1)]$, or $\operatorname{Cap}\left(A_{\tau(k)}\right) \geq c_{2} n / M$. Then for $k \in\left[\frac{1}{4} n, \frac{1}{2} n\right]$, the $X_{k}$ 's stochastically dominate i.i.d. Bernoulli random variables with mean $5(\log n) / n$, and so

$$
\mathbb{P}\left(\sum_{n / 4}^{n / 2} X_{k}<\log n\right)<C e^{-c \log n}=C n^{-c}
$$

However, if $\sum_{n / 4}^{n / 2} X_{k} \geq \log n$ then either $\log n$ splits occur or else at some time $\operatorname{Cap}\left(A_{t}\right)$ exceeded $c_{2} n / M$. In the latter case monotonicity $\operatorname{implies} \operatorname{Cap}\left(A_{\tau}\right)>$ $c_{2} n / M$.

\subsection{Growth estimates}

We shall need the following estimates for the growth rate of the DLA and SDLA.

Lemma 20. $\mathbb{P}\left(\tau(n / \log n)>2 M\right.$ and $\left.\sum_{k=1}^{n / \log n} \frac{1}{\operatorname{Cap}\left(A_{\tau(k)}\right)} \leq M\right)<C n^{-c}$.

Since by definition $\sum \frac{1}{\operatorname{Cap}\left(A_{\tau(k)}\right)}$ is quite likely to be less than $M$, it follows that $\tau(n / \log n)$ is usually less than $2 M$. Before we start with the proof of lemma 20 let us note the following

Lemma 21. Let $X_{1}, \ldots, X_{N}$ be independent Poisson clocks (not necessarily with the same rates). Let $T$ be the first time one of them activated, and let $E$ be the event that the first to activate was $X_{1}$. Then $T$ conditioned on $E$ has the same distribution as $T$.

Proof. This is a straightforward calculation and we omit the details.

Proof of lemma 20. Points are added to $A_{t}$ at rate $\operatorname{Cap}\left(A_{t}\right)$. Let $X_{m}=\tau(m+1)-$ $\tau(m)$ be the time it takes to add the $m+1^{\text {st }}$ point. Let $Q_{m}=\operatorname{Cap}\left(A_{\tau(m)}\right)$ be the rate at which it is added, and $\mathscr{F}$ the sigma-field generated by all $Q_{m}$ 's. Examine one $X_{m}$ conditioned on $\mathscr{F}$. The conditioning gives us the capacity of $A_{m}$ as well as that of $A_{m+1}$ which gives some information on the point that was activated to increase $A_{m}$. Nevertheless, by lemma 21 this information is irrelevant and we get that $X_{m}$ conditioned on $\mathscr{F}$ is an exponential random variable with mean $1 / Q_{m}$. Clearly, conditioning on $\mathscr{F}$ makes the different $X_{m}$-s independent. Thus their sum can be analyzed by standard techniques as follows.

We have that

$$
\mathbb{E}\left(e^{X_{m} / 2} \mid \mathscr{F}\right)=\frac{Q_{m}}{Q_{m}-1 / 2}=1+\frac{1}{2 Q_{m}-1} \leq e^{1 /\left(2 Q_{m}-1\right)} .
$$

Since $Q_{m} \geq 1$, it follows that

$$
\mathbb{E}\left(e^{\left(X_{m}-1 / Q_{m}\right) / 2} \mid \mathscr{F}\right) \leq e^{1 / 2 Q_{m}^{2}}
$$

By Lemma 7 we have $Q_{m}=\operatorname{Cap}\left(A_{\tau_{m}}\right) \geq c m^{1-\alpha}$, so $\sum_{m} \frac{1}{2 Q_{m}^{2}}<C$, and so

$$
\mathbb{E} \exp \left(\frac{1}{2} \sum_{m<n / \log n} X_{m}-\frac{1}{Q_{m}}\right) \leq C
$$

(note that we integrated $\mathscr{F}$ away). Since $\tau(n / \log n)=\sum_{m<n / \log n} X_{m}$ we find

$$
\mathbb{P}\left(\tau(n / \log n)>M+\sum \frac{1}{Q_{m}}\right) \leq C e^{-M / 2}<C n^{-c}
$$


(using $M>c \log n$ ). The claim follows.

Lemma 22. With probability at least $\frac{1}{2}-C n^{-c}$ one has that

$$
\tau(n / \log n) \leq \min \left\{2 M, \beta_{1}\right\}
$$

where $\beta_{1}$ is the time of the first split.

Proof. By lemma 20 and the definition of $M$,

$$
\mathbb{P}(\tau(n / \log n) \leq 2 M) \geq \frac{5}{6}-C n^{-c} .
$$

By lemma 18

$$
\mathbb{P}\left(\beta_{1} \geq \min \{2 M, \tau(n / \log n)\}\right) \geq \frac{2}{3} .
$$

When both events happen (which happens with probability $\geq \frac{1}{2}-\mathrm{Cn}^{-c}$ ) we get the required inequality.

Recall that for some fixed $q, \sigma(m)$ is the (continuous) time that the size of the SDLA (namely $\left|S_{t}\right|+\left|\widehat{S}_{t}\right|$ ) first reaches $m$.

Lemma 23. Fix $q \in \mathbb{N}$, and consider the event $\mathscr{B}$ that $\sigma(n)-\sigma(n / 2)<2 M$ and $\operatorname{Cap}\left(S_{\sigma(n)}\right)+\operatorname{Cap}\left(\widehat{S}_{\sigma(n)}\right)<n / 5 M$. Then $\mathbb{P}(\mathscr{B})<C e^{-c n}$.

Proof. Note that $\operatorname{Cap}\left(S_{t}\right)\left(\operatorname{resp} . \operatorname{Cap}\left(\widehat{S}_{t}\right)\right)$ is the rate at which points are added to $S_{t}$ (resp. $\left.\widehat{S}_{t}\right)$. Consider the process $N_{t}$ that counts points being added to $S_{t}$ or $\widehat{S}_{t}$ starting at time $\sigma(n / 2)$, stopped at the first time $t$ when $\operatorname{Cap}\left(S_{t}\right)+\operatorname{Cap}\left(\widehat{S}_{t}\right)>\frac{n}{5 M}$. Then $N_{t}$ is stochastically dominated by a Poisson process with intensity $\frac{n}{5 M}$. Up to this stopping time, we have $N_{t}=\left|S_{\sigma(n / 2)+t}\right|+\left|\widehat{S}_{\sigma(n / 2)+t}\right|-n / 2$.

Thus the number of points added within time $2 M$ and before the stopping time is dominated by a $\operatorname{Poi}\left(2 M \cdot \frac{n}{5 M}\right)$ variable. The probability that this exceeds $n / 2$ is exponentially small. However, if $N_{2 M}<n / 2$ then either $\sigma(n)-\sigma(n / 2)>2 M$ or else $\operatorname{Cap}\left(S_{t}\right)+\operatorname{Cap}\left(\widehat{S}_{t}\right)$ exceeds $\frac{n}{5 M}$ before time $\sigma(n)$. The claim follows by monotonicity of capacity.

\subsection{Interaction probabilities}

We now analyze the probability of an interaction between $S^{q}$ and $\widehat{S}^{q}$. The bounds we get will imply that for any given $q$, the law of the DLA $A_{t}$ is close to the law of $S_{t} \cup \widehat{S}_{t}$. Fix some $q \in \mathbb{N}$, and consider the SDLA. The set $S_{t} \cup \widehat{S}_{t}$ evolves very similarly to a DLA. The difference is that if a random walk from $S_{t}$ hits $\widehat{S}_{t}$ or vice versa, then a point may be added to the SDLA but not to the DLA. Thus we need to bound the probability of such an intersection happening.

We shall be interested in the union of all random walk trajectories that originate from a point of $S^{q}$ or $\widehat{S}^{q}$ up to time $t$. More precisely, $T_{t}^{q}$ is the union of all trajectories of random walks that started from points of $S^{q}$ up to time $t$ and escaped (i.e. led to the addition of a point to $S^{q}$ ). Similarly, $\widehat{T}_{t}^{q}$ is the union of all trajectories of random walks that started from points of $\widehat{S}^{q}$ up to time $t$ and escaped. However, the walk at time $\beta_{q}$ is treated differently. Even though it starts at a point of $\widehat{S}^{q}$, its first step is to $b_{q} \in S^{q}$, and we include this walk minus its starting point in $T^{q}$ and not in $\widehat{T}^{q}$. Note that paths in both $T^{q}$ and $\widehat{T}^{q}$ include their starting points, so $S^{q} \subset T^{q}$ and $\widehat{S}^{q} \subset \widehat{T}^{q}$. 
Our goal in this section is the following:

Lemma 24. For any $q \in \mathbb{N}$,

$$
\mathbb{P}\left(T_{\sigma}^{q} \cap \widehat{S}_{\sigma}^{q} \neq \emptyset\right) \leq C n^{-c} \quad \text { and } \quad \mathbb{P}\left(\widehat{T}_{\sigma}^{q} \cap S_{\sigma}^{q} \neq \emptyset\right) \leq C n^{-c} .
$$

We first argue that after a large jump the walk is unlikely to hit any given point.

Lemma 25. Consider an $\alpha$-random walk $R$ started at $a$, and a point $z$, and let $R_{1}$ be the first jump of the random walk. Then $\mathbb{P}_{a}\left(R\right.$ hits $\left.z,\left|R_{1}-a\right|>L\right) \leq C / L$.

Consequently, for any fixed set $S$, the probability of making a jump of size at least $L$ and hitting $S$ is at most $C|S| / L$.

Proof. With $R_{1}=a+X$, we use that Green's function satisfies $G\left(z-R_{1}\right) \approx$ $\left|z-R_{1}\right|^{\alpha-1}$ (lemma 2) to get

$$
\mathbb{P}_{a}\left(\left|R_{1}-a\right|>L, R \text { hits } z\right) \leq C \sum_{|X|>L}|X|^{-\alpha-1}|z-a-X|^{\alpha-1} .
$$

In this last sum, terms with $|z-a-X| \leq L$ are bounded by $\sum_{-L}^{L} L^{-\alpha-1}|i|^{\alpha-1} \leq$ $C L^{-1}$. Terms with $|z-a-X|>L$ are bounded by $\sum_{|X|>L}|X|^{-\alpha-1} L^{\alpha-1} \leq C / L$ as well.

Recall that $b_{q}$ is the birth point, the first point in $S^{q}$. By $A-x$ we denote the translation of a set $A$ by $x$.

Lemma 26. Let $T$ be either $T_{\tau^{q}(n)}^{q}-b_{q}$ or $\widehat{T}_{\widehat{\tau}^{q}(n)}^{q}$ for some $q \in \mathbb{N}$, and let $I$ be any interval of length $L \geq D$. Then

$$
\mathbb{E}|T \cap I| \leq C n L^{\alpha} .
$$

Roughly, this is so since we are considering $n$ random walks, and each of those visits $L^{\alpha}$ points in $I$. This is somewhat complicated by the fact that there are also random walks that do not escape $S^{q}$ but still visit $I$.

Proof. We may assume (by increasing $C$ if necessary) that $L$ is sufficiently large. We consider only the case of $T^{q}$, the case of $\widehat{T}^{q}$ is proved identically. We show the stronger fact, that over the time it takes to add a single point to $S^{q}$ the expected number of points visited in $I$ is at most $C L^{\alpha}$.

Examine the random walk at some time when it is in $I$. The random walks we are dealing with have probability at least $c L^{-\alpha}$ to make a step bigger than $2 L$. By lemma 6 once this happens it has probability bigger than some $c$ to never hit $I$ again, hence it has probability at least $c L^{-\alpha}$ to make a large step and then never hit $I$ again.

Next apply the previous lemma. We get that (again, after the initial step bigger than $2 L$ ) the probability that the random walk hits $S^{q}$ is at most $C\left|S^{q}\right| / L$. Since $\left|S^{q}\right| \leq n$ and since $L \geq D \geq c n^{1 / \alpha}$ (by (17)) we get that $C\left|S^{q}\right| / L \leq C L^{\alpha-1} \ll L^{-\alpha}$ (here we only need $\alpha<\frac{1}{2}$ ). Hence we get, for $L$ sufficiently large, that with probability at least $c L^{-\alpha}$ the random walk makes a step bigger than $2 L$ and after that disappears to infinity, never returning to either $I$ or $S^{q}$. This, of course, adds a point to $S^{q}$.

Since all these calculations were independent of the past, we get that the number of points visited in $I$ before adding a single point to $S^{q}$ is stochastically dominated by a geometric random variable with expectation $C L^{\alpha}$, proving the lemma. 
Lemma 27. Let $T$ be either $T_{\tau^{q}}^{q}-b_{q}$ or $\widehat{T}_{\bar{\tau}^{q}}^{q}$ for some $q \in \mathbb{N}$. Fix any $x \in \mathbb{Z}$, and let $\nu$ be an independent random walk step conditioned on $|\nu| \geq D$. Then

$$
\mathbb{P}(x-\nu \in T) \leq C n D^{\alpha-1} \leq C n^{2-1 / \alpha} .
$$

Proof. Let $I_{k}=\left[2^{k} D, 2^{k+1} D\right)$. Then we can write

$$
\mathbb{P}(x-\nu \in T)=\sum_{k \geq 0} \mathbb{P}\left(\nu \in I_{k}, x-\nu \in T\right)+\sum_{k \geq 0} \mathbb{P}\left(-\nu \in I_{k}, x-\nu \in T\right) .
$$

Since $\mathbb{P}(\nu=y) \leq C D^{\alpha} y^{-\alpha-1}$ (the $D^{\alpha}$ comes from the conditioning of $\nu$ to be large), we have for $y \in I_{k}$ that $\mathbb{P}(\nu=y) \leq C 2^{-(\alpha+1) k} D^{-1}$. Thus

$$
\begin{aligned}
\mathbb{P}\left(\nu \in I_{k}, x-\nu \in T\right) & =\sum_{y \in I_{k}} \mathbb{P}(\nu=y) \mathbb{P}(x-y \in T) \\
& \leq C 2^{-(\alpha+1) k} D^{-1} \sum_{y \in I_{k}} \mathbb{P}(x-y \in T) \\
& =C 2^{-(\alpha+1) k} D^{-1} \mathbb{E}\left|T \cap\left(x-I_{k}\right)\right| \\
\text { By Lemma 26 } & \leq C 2^{-(\alpha+1) k} D^{-1} n\left|I_{k}\right|^{\alpha}=C 2^{-k} n D^{\alpha-1} .
\end{aligned}
$$

The same bound holds for $-\nu \in I_{k}$. Summing over $k$ now gives $\mathbb{P}(x-\nu \in T) \leq$ $C n D^{\alpha-1}$. The last claim holds since $D>c n^{1 / \alpha}(17)$.

We remark that this last lemma is the most important point in the proof where we need a pointwise estimate on $\mathbb{P}(\nu=y)$ and cannot do with an estimate on $\mathbb{P}(\nu>y)$.

Proof of Lemma 24. We prove only the first bound, as the second is proved identically.

Let $\nu$ be the jump of the walk the creates $S^{q}$ i.e. the $q^{\text {th }}$ large jump. Let $a$ be the point of $\widehat{S}^{q}$ from which the jump occurred (so $b_{q}=a+\nu$ ). The first key observation is that $\nu$ is independent of $\widehat{S}^{q}$, and only affects $S^{q}$ by a translation, so it is independent also of $S^{q}-\nu$.

We have that $S_{t}^{q}-a-\nu$ and $\widehat{S}_{t}^{q}$ are also independent for any $t$. Note that for the stopping time $\sigma$, the independence fails, since their sizes are now linked. To overcome this, we consider intersections among the larger sets $\widehat{S}_{\widehat{\tau}^{q}}^{q}$ and $T_{\tau^{q}}^{q}$. For those we have that $\widehat{S}_{\widehat{\tau}^{q}}^{q}, T_{\tau^{q}}^{q}-a-\nu$ and $\nu$ are jointly independent, with $\widehat{S}_{\widehat{\tau}^{q}}^{q}$ being some set of size $n$ and $\nu$ being a random walk step, conditioned to be large. Moreover, $a$ depends only on $\widehat{S}_{\widehat{\tau}^{q}}^{q}$.

Denote $S=\widehat{S}_{\widehat{\tau}^{q}}^{q}$ and $T=T_{\tau^{q}}^{q}-a-\nu$, so we are interested in the probability that $S-a-\nu$ intersects $T$. Condition on $a, S$, and consider any $s \in S$. By Lemma 27 we have that $\mathbb{P}(s-a-\nu \in T) \leq C n^{2-1 / \alpha}$. Since $|S|=n$ it follows that $\mathbb{P}(S-a-\nu \cap T \neq \emptyset) \leq C n^{3-1 / \alpha}$. Since this bound is uniform in $S$ and $a$, and since $\alpha<1 / 3$, this completes the proof.

The last part of this proof is the only place where $\alpha<\frac{1}{3}$ is crucial to our proof. It is also used in the next lemma, though a weaker statement which should hold for $\alpha<\frac{1}{2}$ would suffice there. However, Lemma 24 fails for $\alpha \in\left[\frac{1}{3}, \frac{1}{2}\right]$, as we expect there to be intersections. Using a weaker form of this lemma will probably require significant modification of our argument. 


\subsection{Bounds on capacity influence}

Let us extend the definition of the Green's function to sets $A, B \subset \mathbb{Z}$ by

$$
G(A, B)=\sum_{\substack{a \in A \\ b \in B}} G(a, b) .
$$

The reason for this is the easy bound $\operatorname{Cap}(A \cup B) \geq \operatorname{Cap}(A)+\operatorname{Cap}(B)-G(A, B)$, which holds since the capacity is the sum of the escape probabilities. With this in mind we prove the following.

Lemma 28. For any $q \in \mathbb{N}$ we have $\mathbb{E} G\left(S_{\tau}^{q}, \widehat{S}_{\tau}^{q}\right) \leq C$.

Proof. Since $\tau_{q}, \widehat{\tau}_{q} \geq \tau$ it is enough to prove that $\mathbb{E} G\left(S_{\tau_{q}}^{q}, \widehat{S}_{\widehat{\tau}_{q}}^{q}\right) \leq C$, i.e. allow each of the two sets to continue to grow to size $n$.

As before, let $\nu$ be the size of the $q^{\text {th }}$ large jump that gave rise to $S^{q}$. Denote $A=\widehat{S}_{\widehat{\tau}_{q}}^{q}$ and $B=S_{\tau_{q}}^{q}-\nu$, and note that $A, B$ are sets of size $n$ and $\nu$ a random walk step conditioned to be large, independent of $A, B$ (the independence claim is as in the previous lemma). Conditioning on $A, B$ we get

$$
\mathbb{E} G\left(S_{\tau^{q}}^{q}, \widehat{S}_{\widehat{\tau}^{q}}^{q}\right)=\sum_{\substack{x \in A \\ y \in B}} \mathbb{E} G(x-(y+\nu))
$$

where the expectations are only over $\nu$. Recall that $G(x) \approx|x|^{\alpha-1}$ by lemma 2 and that $\mathbb{P}(\nu=z)<C / D$ for all $z$. Therefore for any $x$ we have

$$
\begin{aligned}
\mathbb{E} G(x-\nu) & \leq \sum_{y:|x-y| \leq D} C|x-y|^{\alpha-1} \cdot \mathbb{P}(\nu=y)+C D^{\alpha-1} \mathbb{P}(|\nu-x|>D) \\
& \leq \frac{C}{D} \sum_{i=1}^{D} i^{\alpha-1}+C D^{\alpha-1} \leq C D^{\alpha-1} \stackrel{(17)}{\leq} C n^{1-1 / \alpha} .
\end{aligned}
$$

Summing over the sets $A$ and $B$ gives

$$
\mathbb{E} G\left(S_{\tau_{q}}^{q}, \widehat{S}_{\widehat{\tau}_{q}}^{q}\right) \leq C n^{3-1 / \alpha} \leq C
$$

\subsection{Coupling DLA and SDLA}

We now have in place all the necessary estimates about DLA and SDLA. In order to put them to use we need to describe a coupling between the processes. More precisely, we construct the DLA as well as all $q$-SDLAs (for all $q \in \mathbb{N}$ ) in the same probability space. This coupling will satisfy the following two properties, which we formulate as lemmas:

Lemma 29. If for some $q$ and $t$ we have $T_{t}^{q} \cap \widehat{S}_{t}^{q}=\widehat{T}_{t}^{q} \cap S_{t}^{q}=\emptyset$, then $A_{t}=S_{t}^{q} \cup \widehat{S}_{t}^{q}$ (as a disjoint union).

Recall that $T^{q}$ is the union of all walks used to create $S^{q}$ as defined in the beginning of $\S 4.5$. Recall also that $\beta_{q}$ is the time the $q^{\text {th }}$ split occurs (i.e. that $S^{q}$ is created), that $b_{q}$ is the first point in $S^{q}$, and and that $\zeta_{q}$ is the first time a split occurs from a point of $S^{q}$.

Lemma 30. The processes $\left(\left\{S_{t+\beta_{q}}^{q}\right\}-b_{q}: t \leq \zeta_{q}-\beta_{q}\right)$, i.e. $S^{q}$ killed at its first split, form an i.i.d. sequence. Each has the law of DLA killed once a random walk begins with a large jump. 
Thus we have on the one hand that the SDLAs for different $q$ are independent until they first split, and on the other hand they are all part of the large DLA process to the extent possible.

The coupling is constructed as follows. Start with the DLA process $A_{t}$. For any $q \in \mathbb{N}$, the $q$-SDLA has $\widehat{S}_{t}^{q}=A_{t}$ until the $q^{\text {th }}$ time a random walk starts with a large jump, at which time $S_{t}^{q}$ becomes non-empty. As long as $A_{t}=S_{t}^{q} \cup \widehat{S}_{t}^{q}$, there is a natural way to continue the coupling: the same points are activated in both processes and the same random walks used from active points. As long as $T_{t}^{q} \cap \widehat{S}_{t}^{q}=\widehat{T}_{t}^{q} \cap S_{t}^{q}=\emptyset$, points are added to $A_{t}$ if and only if they are also added to one of $S_{t}^{q}$ or $\widehat{S}_{t}^{q}$.

This guarantees that Lemma 29 holds, regardless of how $S^{q}$ and $\widehat{S}^{q}$ evolve once there is some interaction between them. In order to achieve the independence property of Lemma 30, we say that once $T_{t}^{q} \cap \widehat{S}_{t}^{q} \neq \emptyset$ or $\widehat{T}_{t}^{q} \cap S_{t}^{q} \neq \emptyset$, the $q$ SDLA continues its evolution independently of the DLA and all other $q^{\prime}$-SDLAs. Lemma 30 now holds since the point activation and associated random walks used to generate $S^{q}$ over the time interval $\left[\beta_{q}, \zeta_{q}\right]$ are all independent and disjoint of those used for any other $q$.

To convince oneself that the activations and random walks are really disjoint, colour $A_{t}$ as follows: the first point is coloured 0 , and whenever a point $a$ is activated and the random walk adds a new point $b$ to the DLA, $b$ inherits $a$ 's colour, except when a split occurs, in which case, if this is the $q^{\text {th }}$ split, $b$ gets coloured by $q$ (the "colours" are elements of $\{0,1,2, \ldots\})$. It is easy to check that $S^{q}$ is exactly the points that have colour $q$ until one of the two events happen:

- either $T^{q} \cap \widehat{S}^{q} \neq \emptyset$ happened, which is one of the two "bad" events of lemma 29, so after that $S^{q}$ becomes independent of everything else,

- or $\zeta_{q}$ occurs, in which case a split happens from $S^{q}$ and $S^{q}$ now corresponds to two colours in $A$.

This shows that the portions of $S^{q}$ during $\left[\beta_{q}, \zeta_{q}\right]$ are independent, since their randomness either comes from differently coloured points, or from a completely independent source.

Now that lemmas 29 and 30 are proved, we have all the necessary pieces.

\subsection{The grand assembly}

We are now ready to put together all the parts of the argument, and prove Theorem 17. The next lemma is the core of the proof. It uses everything we learned so far, and the theorem follows from it by a simple induction. Recall that $\operatorname{Med}\left(\cdot ; \frac{1}{6}\right)$ is the $\frac{1}{6}$-quantile defined in (15).

Lemma 31. For some $c_{3}$, with probability at least $1-C n^{-c}$,

$$
\operatorname{Cap}\left(A_{n}\right) \geq \min \left\{\frac{c_{3} n}{M}, \frac{\log n}{5} \operatorname{Med}\left(\operatorname{Cap}\left(A_{n / \log n}\right) ; \frac{1}{6}\right)\right\} .
$$

Proof. We may assume $n$ is sufficiently large, as for small $n$ the lemma is true trivially if the $C$ in the probability estimate $1-C n^{-c}$ is made large enough. For every $q \in \mathbb{N}$ Let $S^{q}$ be a $q$-SDLA process, and assume that they are all coupled to our DLA as in the previous section. With high probability the (bad) event of Lemma 19 does not occur for the DLA and the (bad) events of Lemmas 23 and 24 do not occur for any $q \leq \log n$. Here and below "with high probability" means 
with probability at least $1-C n^{-c}$. To spare the reader some page flipping here is a short reminder of these lemmas (up to the aforementioned bad events):

Lemma 19 Either the DLA splits $\log n$ times or it has large capacity.

Lemma 23 Either the SDLA satisfies $\sigma(n)-\sigma(n / 2) \geq 2 M$ or it has large capacity.

Lemma $24 T \cap \widehat{S}=\widehat{T} \cap S=\emptyset$.

Thus we suppose from here on that this is the case. Recall that $\tau$ is the stopping time when the DLA reaches size $n$ and $\sigma$ is the analogous quantity for the $q$-SDLA. From Lemma 29 we now see that $\sigma(m)=\tau(m)$ for any $m \leq n$ and for any $q \leq \log n$.

If for some $q \leq \log n$ we have $\operatorname{Cap}\left(S_{\sigma}\right)+\operatorname{Cap}\left(\widehat{S}_{\sigma}\right) \geq n / 5 M$ then at least one of them has capacity at least $n / 10 M$. Since $A_{\tau}=S_{\sigma} \cup \widehat{S}_{\sigma}$ (again using Lemma 29) by the monotonicity of capacity we get $\operatorname{Cap}\left(A_{\tau}\right) \geq n / 10 M$ and we are done as long as $c_{3} \leq \frac{1}{10}$. Thus suppose this too is not the case, and so, since the bad event of Lemma 23 did not occur, we get $\tau(n)-\tau(n / 2) \geq 2 M$. By making $c_{3}<c_{2}$ (with $c_{2}$ taken from Lemma 19) we may similarly assume (this time using Lemma 19) that $A$ splits at least $\log n$ times by time $\tau(n / 2)$.

Call a split $S^{q}$ good if the following both hold:

(i) its capacity is typical: $\operatorname{Cap}\left(S_{\tau_{q}(n / \log n)}^{q}\right) \geq \operatorname{Med}\left(\operatorname{Cap}\left(A_{\tau(n / \log n)}\right) ; \frac{1}{6}\right)$, and

(ii) it grows fast enough: $\tau_{q}(n / \log n)<\min \left\{\beta_{q}+2 M, \zeta_{q}\right\}$, that is, $S^{q}$ reaches size $n / \log n$ both before splitting $\left(\zeta_{q}\right)$ and within $2 M$ of becoming nonempty $\left(\beta_{q}\right)$.

Since this only depends on the process $S^{q}$ until it splits, the events that $S^{q}$ are good are i.i.d. (by Lemma 30). By the definition of Med, the probability of clause (i) holding for any $q$ is at least $\frac{5}{6}$. On the other hand, Lemma 22 gives that clause (ii) holds with probability at least $\frac{1}{2}-C n^{-c}$. Hence each $S^{q}$ is good with probability at least $\frac{1}{3}-C n^{-c}$. Thus (for $n$ large enough) the probability that at least $\frac{1}{4} \log n$ of the $S^{q}$ for $q \leq \log n$ are good is at least $1-C e^{-c \log n}=1-C n^{-c}$. Assume this is the case.

To summarize our current assumptions, either $\operatorname{Cap}\left(A_{\tau}\right)>\frac{c_{3} n}{M}$, or else there are at least $\frac{1}{4} \log n$ good branches $S^{q}$ with $q<\log n$, each of which started before time $\tau(n / 2)$ and accumulated $n / \log n$ points without splitting and within time $2 M$. Since also $\tau(n)-\tau(n / 2) \geq 2 M$, these points are all present in $A_{\tau}$ and separate from those of other good branches. Moreover, the first $n / \log n$ points in each of these branches have a typically large capacity.

Let $\mathscr{G}=\left\{q<\log n, S^{q}\right.$ is good $\}$, so that $A_{\tau}$ contains $\bigcup_{q \in \mathscr{G}} S_{\tau_{q}(n / \log n)}^{q}$. This implies the capacity bound

$$
\operatorname{Cap}\left(A_{\tau}\right) \geq \sum_{q \in \mathscr{G}} \operatorname{Cap}\left(S_{\tau_{q}(n / \log n)}^{q}\right)-C \sum_{q \in \mathscr{G}} G\left(S_{\tau}^{q}, \widehat{S}_{\tau}^{q}\right) .
$$

(In fact, it suffices to subtract the Green's function between $S_{\tau_{q}(n / \log n)}^{q}$ and the union of that set for other good $q$ 's, which is smaller.) Now, for each good $S^{q}$ we have $\operatorname{Cap}\left(S_{\tau_{q}(n / \log n)}^{q}\right) \geq \operatorname{Med}\left(\operatorname{Cap}\left(A_{\tau(n / \log n)}\right) ; \frac{1}{6}\right)$, and each term in the second sum has bounded expectation (by Lemma 28). It follows that

$$
\mathbb{E} \sum_{q \in \mathscr{G}} G\left(S_{\tau}^{q}, \widehat{S}_{\tau}^{q}\right) \leq \mathbb{E} \sum_{q=1}^{\log n} G\left(S_{\tau}^{q}, \widehat{S}_{\tau}^{q}\right) \leq C \log n
$$


and so this sum exceeds $n^{c}$ only with probability $C n^{-c} \log n$. If it does not then because $\operatorname{Cap}\left(A_{\tau(n / \log n)}\right) \geq c(n / \log n)^{1-\alpha}($ Lemma 7$)$,

$$
\begin{aligned}
\operatorname{Cap}\left(A_{\tau}\right) & \geq \frac{\log n}{4} \operatorname{Med}\left(\operatorname{Cap}\left(A_{\tau(n / \log n)}\right) ; \frac{1}{6}\right)-C n^{c} \\
& \geq \frac{\log n}{5} \operatorname{Med}\left(\operatorname{Cap}\left(A_{\tau(n / \log n)}\right) ; \frac{1}{6}\right)
\end{aligned}
$$

(for $c$ small and $n$ large). This completes the proof.

Lemma 32. $M_{n}=n^{o(1)}$, and $\operatorname{Med}\left(\operatorname{Cap} A_{\tau}(n) ; \frac{1}{6}\right)=n^{1-o(1)}$.

Proof. We now no longer need the SDLA process, so to keep notations clear, denote $Q_{n}=\operatorname{Cap}\left(A_{\tau(n)}\right)$. All we need about these random variables is that $Q_{1}=1$, $Q_{n+1} \geq Q_{n}$, that $M_{n}$ is (by definition) the $\frac{5}{6}$-median of $\sum^{n} Q_{i}^{-1}$, and that with high probability

$$
Q_{n} \geq \min \left\{\frac{c_{3} n}{M_{n / \log n}}, \frac{\log n}{5} \operatorname{Med}\left(Q_{n / \log n} ; \frac{1}{6}\right)\right\} .
$$

In particular, for some $\ell_{0}$, (18) holds simultaneously for all $n=2^{\ell}, \ell \geq \ell_{0}$ with probability at least $\frac{5}{6}$. Call the event that this happens $\mathscr{G}$.

Fix $\varepsilon>0$, and make $\ell_{0}$ larger if needed, so that $\left(\log 2^{\ell_{0}}\right)^{\varepsilon}>\max \left\{2 / c_{3} \varepsilon, 10\right\}$. (This can only increase $\mathscr{G}$.) We now pick some $K=K\left(\ell_{0}\right)$ sufficiently large such that for all $n \leq 2^{\ell_{0}}$

$$
M_{n} \leq K n^{\varepsilon}, \quad \text { and } \quad Q_{n} \geq \frac{n^{1-\varepsilon}}{K \varepsilon}
$$

We now prove by induction that on the event $\mathscr{G},(19)$ holds for all $n$ (the left clause in (19) is just an inequality of numbers so we just show that it holds - the right clause is an event, and we show that it follows from $\mathscr{G})$.

To see this, consider some $n>2^{\ell_{0}}$, and let $n^{\prime}=2^{\ell} \leq n$ be the largest power of 2 below $n$. We first show the right clause in (19). Since $Q_{n}$ is monotone, applying (18) with $n^{\prime}$ (which we are allowed, because we assume the event $\mathscr{G}$ happened) we get either

$$
Q_{n} \geq \frac{c_{3} n^{\prime}}{M_{n^{\prime} / \log n^{\prime}}} \quad \text { or } \quad Q_{n} \geq \frac{\log n^{\prime}}{5} \operatorname{Med}\left(Q_{n^{\prime} / \log n^{\prime}} ; \frac{1}{6}\right) .
$$

Under the induction hypothesis (19) for $n^{\prime} / \log n^{\prime}$, the former case implies

$$
Q_{n} \geq \frac{c_{3} n^{\prime}}{M_{n^{\prime} / \log n^{\prime}}} \geq \frac{c_{3} n^{\prime}}{K\left(n^{\prime} / \log n^{\prime}\right)^{\varepsilon}} \geq \frac{c_{3}}{2 K} n^{1-\varepsilon}\left(\log n^{\prime}\right)^{\varepsilon}
$$

By our assumption that $\left(\log 2^{\ell_{0}}\right)^{\varepsilon}>2 / c_{3} \varepsilon$, this case yields $Q_{n}>n^{1-\varepsilon} /(K \varepsilon)$, as needed. For the latter case in $(20)$ note that because $\mathbb{P}(\mathscr{G})>\frac{5}{6}$, the induction hypothesis implies that $\operatorname{Med}\left(Q_{m} ; \frac{1}{6}\right) \geq m^{1-\varepsilon} /(K \varepsilon)$ for all $m<n$ and in particular for $n^{\prime} / \log n^{\prime}$ so we get

$$
Q_{n} \geq \frac{\log n^{\prime}}{5} \operatorname{Med}\left(Q_{n^{\prime} / \log n^{\prime}} ; \frac{1}{6}\right) \geq \frac{\log n^{\prime}}{5} \frac{\left(n^{\prime} / \log n^{\prime}\right)^{1-\varepsilon}}{K \varepsilon} \geq \frac{1}{10 K \varepsilon} n^{1-\varepsilon}\left(\log n^{\prime}\right)^{\varepsilon} .
$$

By our assumption that $\left(\log 2^{\ell_{0}}\right)^{\varepsilon}>10$ this case too yields $Q_{n}>n^{1-\varepsilon} /(K \varepsilon)$. 
It remains to bound $M_{n}$. This is easy, since on the event $\mathscr{G}$ we have $Q_{i} \geq \frac{i^{1-\varepsilon}}{K \varepsilon}$ for all $i \leq n$. On this event,

$$
\sum_{i=1}^{n} Q_{i}^{-1} \leq K \varepsilon \sum_{i=1}^{n} i^{\varepsilon-1} \leq K n^{\varepsilon}
$$

Since $\mathscr{G}$ has probability bigger than $\frac{5}{6}$, we get that the $\frac{5}{6}$-median of the sum, namely $M_{n}$ is at most $K n^{\varepsilon}$. This completes the induction and shows that under $\mathscr{G}$, (19) holds for all $n$. Since $\varepsilon$ was arbitrary, the lemma is proved.

Proof of Theorem 1\%. By monotonicity we may consider only $n=2^{\ell}$. By Lemmas 32 and 31, $\mathbb{P}\left(\operatorname{Cap}\left(A_{n}\right)>n^{1-o(1)}\right)>1-C n^{-c}$. By Borel-Cantelli, this event holds for all but finitely many such $n$.

\section{Acknowledgements}

We would like to thank Itai Benjamini for initiating this project and for many enlightening discussions. GA and GK's work partially supported by the Israel Science Foundation. OA's work partially supported by the NSERC and the ENS.

\section{References}

[AABK] Gideon Amir, Omer Angel, Itai Benjamini and Gady Kozma, One-dimensional longrange diffusion-limited aggregation I. Preprint (2009), available from arxiv:0910.4416

[A] Gideon Amir, One-dimensional long-range diffusion-limited aggregation III - The limit aggregate. Preprint (2009), available from arxiv:0911.0122

[BL02] Richard F. Bass and David A. Levin, Transition Probabilities for Symmetric Jump Processes. Trans. Amer. Math. Soc. 354 (2002), 2933-2953. Available at: ams.org

[B99] Daniel Bertilsson, On Brennan's conjecture in conformal mapping. Ph.D. Thesis, Department of Mathematics, Rotal Institute of Technology, Stockholm, Sweden.

[C85] Lennart Carleson, On the support of harmonic measure for sets of Cantor type, Ann. Acad. Sci. Fenn. Ser. A I Math. 10 (1985), 113-123.

[GR91] Jean-François Le Gall and Jay Rosen, The range of stable random walks, Ann. Probab. 19:2 (1991), 650-705. Available at: projecteuclid.org

[HJ85] Roger A. Horn and Charles R. Johnson Matrix Analysis, Corrected reprint of the 1985 original. Cambridge University Press, Cambridge, 1990. xiv+561 pp. ISBN: 0-521-38632-2, $15-01$

[JW88] Peter W. Jones and Thomas H. Wolff, Hausdorff dimension of harmonic measures in the plane, Acta Math. 161:1-2 (1988), 131-144. Available at: springerlink.com

[M89] Nikolai G. Makarov, Вероятностные методы в теории конформных отображений [Russian: Probability methods in the theory of conformal mappings], Algebra i Analiz 1:1 (1989), 3-59. Available at: mathnet.ru. English translation in Leningrad Math. J. 1:1 (1990), 1-56.

[M98] Nikolai G. Makarov, Fine structure of harmonic measure, Algebra i Analiz 10:2 (1998), 1-62. Available at: mathnet.ru

[S76] Frank Spitzer, Principles of random walks. Second edition. Graduate Texts in Mathematics, Vol. 34. Springer-Verlag, New York-Heidelberg, 1976. xiii +408 pp.

[W68] John A. Williamson, Random walks and Riesz kernels. Pacific J. Math. 25:2 (1968) 393415. Available at: projecteuclid.org

$$
\begin{array}{r}
\text { Gideon Amir } \\
\text { gidi.amir@gmail.com } \\
\text { Bar-Ilan University, Ramat Gan, Israel } \\
\text { Omer Angel } \\
\text { angel@math.ubc.ca } \\
\text { University of British Columbia, Vancouver, Canada }
\end{array}
$$


Gady Kozma gady. kozma@weizmann. ac.il The Weizmann Institute of Science, Rehovot, Israel 